\title{
SOME MEANS OF CONVEX BODIES $\left({ }^{1}\right)$
}

BY

W. J. FIREY

The systematic study of various mean-values of nonnegative valued functions is due in large part to the work of J. L. W. V. Jensen [12], A. Kolmogoroff [11], and B. Jessen [13]. In this paper we present a similar theory of certain means of functions whose values are taken from a collection of convex bodies in a finite dimensional Euclidean space. The range of nonnegative valued functions is totally ordered while the collection of convex bodies to be considered is partially ordered by set inclusion; corresponding to inequalities, we shall have inclusions, here to be called inequalities, between mean-values. Thus the discussion will furnish an example of a partially ordered system whose algebraic and topological structure is sufficient to admit a fairly elaborate theory of inequalities analogous to that of the real numbers.

The first section is a résumé of those parts of the work of Jensen, Kolmogoroff, and Jessen for which analogues will be developed. $\$ 2$ treats of pertinent material about star and convex bodies. Certain families of star bodies and of convex bodies are defined in the third section; these latter play the role of nonnegative valued functions. Two systems of power means of such families are described. Each system is, in its way, analogous to the power means of nonnegative valued functions. In $\S 4$ we discuss some special cases of power means including elementary means and the Riemann-Minkowski integrals of A. Dinghas [2], [3]. Also some rotation invariants of a convex body are described as power means of special families determined by the convex body. The fifth section begins with crucial properties of the means and contains an extremal characterization for the two systems of means defined in the third section. Analogues of Jensen's and Jessen's inequalities make up $\S 6$. We also mention the limiting cases of power means as the power index becomes infinite, positively and negatively. As an application of Jessen's inequality, a Brunn-Minkowski type theorem is proved. In the final section we discuss some further systems of means of convex bodies.

1. The definition of the elementary power means extends in a natural way to include power means of certain functions $f$ which have finite, nonnegative values and whose domain $T$ is a compact topological group equipped with its Haar measure. If $f$ is bounded and measurable over its domain, its $p$ th power mean is defined by

$$
\sqrt[p]{ } \int_{T} f^{p}(t) d t=M_{p}(f)
$$

Received by the editors March 2, 1964.

( ${ }^{1}$ ) This work was supported in part by a grant from the National Science Foundation, NSF-G19838. 
where the integration is extended over the domain of $f$. It is assumed that the Haar measure is so normalized that if $f(t)=1$ over $T$, we have $M_{1}(f)=1$. We allow the index $p$ to be any number from the set $-\infty \leqq p \leqq+\infty$.

Certain conventions must be adopted in (1.1); these are chosen to preserve special continuity properties of $M_{p}(f)$. For $p=0$ we define

$$
M_{0}(f)=\exp \int_{T} \ln f(t) d t
$$

Similarly we set

$$
M_{\infty}(f)=\operatorname{eff}_{T} \sup f, \quad M_{-\infty}(f)=\underset{T}{\operatorname{effinf}} f .
$$

If $f$ vanishes over a set of positive measure, we define $M_{p}(f)=0$ for $p \leqq 0$.

Two particular domains of definition will be of importance: the closed interval of real numbers $t$ satisfying $0 \leqq t \leqq 1$, to be denoted by $T_{1}$; the group $\mathscr{R}_{n}$ of proper, (i.e., orientation preserving), rotations about the origin of Euclidean $n$-space $R_{n}$. We view $T_{1}$ as a group under the operation of addition modulo one.

The classical inequalities may be described in terms of two inequalities governing power means. First, Jensen's inequality, cf. Jensen [12], valid for any nonnegative valued $f$ which is measurable over $T$ :

$$
M_{p}(f) \leqq M_{q}(f) \text { for } p \leqq q .
$$

So, for example, the choice $p=0, q=1$ gives the inequality of the arithmetic and geometric means.

Second, Jessen's inequality, cf. Jessen [13], valid for any nonnegative valued $f$ which is measurable over $T \times T^{\prime},\left(T^{\prime}\right.$ is also assumed to be a compact, topological group),

$$
M_{q}^{t^{\prime}} M_{p}^{t}(f) \leqq M_{p}^{t} M_{q}^{t^{\prime}}(f) \text { for } p \leqq q .
$$

For Jessen's inequality $M_{q}^{t^{\prime}}(f)$ denotes the function, defined almost everywhere in $T$, by

$$
v^{\prime \prime} \int_{T^{\prime}} f^{q}\left(t, t^{\prime}\right) d t^{\prime}
$$

Thus the formation of the right and left sides of (1.3) requires an appeal to Fubini's theorem in a rather general form, cf. [8, p. 148]. When we choose $p=1$ in (1.3), we obtain the commoner form of Minkowski's inequality; the choice $q=1$ gives the remaining cases.

It is a consequence of work of Kolmogoroff [11] and Nagumo [14] that the means $M_{p}$ for $|p|<\infty$ can be characterized by a few simple properties. More precisely, if $M$ is a functional defined for each nonnegative function $f$ which is bounded and measurable over $T_{1}$, and if $M$ satisfies conditions (1) through (6) below, then for some real, finite $p$ we have $M(f)=M_{p}(f)$ over the domain $D$ of $M$. 
Let $f, f_{i}$, and $g$ denote functions in $D$.

(1) $M$ is continuous; that is, if $\left\{f_{j}\right\}$ is a bounded sequence converging pointwise to $f$ over $T_{1}$, then $M\left(f_{j}\right) \rightarrow M(f)$.

(2) $M$ is monotonic increasing in the sense that if $f(t) \leqq g(t)$ over $T_{1}$, then $M(f) \leqq M(g)$.

(3) $M$ is symmetric; that is if $g(t)=f(t+\tau)$ for some $\tau$ in $T_{1}$, the addition being modulo one, then $M(f)=M(g)$.

(4) If $f(t)=1$ over $T_{1}$, then $M(f)=1$.

(5) Let $T_{1}^{\prime}$ be the subinterval $\tau \leqq t \leqq \tau^{\prime}$ of $T_{1}$ and set $t^{\prime}=(t-\tau) /\left(\tau^{\prime}-\tau\right)$. We define $g$ over $T_{1}$ by setting $g\left(t^{\prime}\right)=f(t)$ for $t$ in $T_{1}^{\prime}$; then we define $f_{1}$ over $T_{1}$ by $f_{1}(t)=M(g)$ for $t$ in $T_{1}^{\prime}, f_{1}(t)=f(t)$ elsewhere. We have $M\left(f_{1}\right)=M(f)$. This will be called the composition property.

(6) $M$ is positively homogeneous of degree one; that is, for every $\lambda>0$, we have $M(\lambda f)=\lambda M(f)$.

In addition to the references cited, the reader is referred to [9] for details.

2. In the Euclidean space $R_{n}$ of points $\left(x^{1}, \ldots, x^{n}\right)$, the point

$$
\left(\lambda x^{1}+\mu y^{1}, \ldots, \lambda x^{n}+\mu y^{n}\right)
$$

$\lambda, \mu$ real, will be written $\lambda x+\mu y$. If $x$ is not the origin 0 , the ray of points of the form $\lambda x, \lambda>0$, will be denoted by $R_{x}$. For the inner product $x^{1} y^{1}+\cdots+x^{n} y^{n}$ we write $(x, y)$ and $\|x\|$ for the distance $(x, x)^{1 / 2}$. E will mean the sphere of points $x$ for which $\|x\| \leqq 1$. If $S$ is a set, $\partial S$ means the boundary of $S$ and $\bar{S}$ means the convex closure of $S$.

Suppose $G$ is a function defined over $R_{n}$ which satisfies:

(a) $G(x)>0$ if $x \neq 0, G(0)=0$;

(b) $G(\lambda x)=\lambda G(x)$ for all $\lambda>0$;

(c) $G$ is continuous for all $x$.

Then $G$ will be called a distance function for the set of points $S$ such that $G(x) \leqq 1$. $S$ is a star body, by which we mean $S$ is closed, bounded, starlike with respect to the origin 0 and has 0 as an interior point. From such a set $S$ we can recover its distance function $G$ by taking $G(0)=0, G(x)=\|x\| /\|z\|$ if $x \neq 0$ where $z=R_{x} \cap \partial S$. Thus the class of distance functions is in one-to-one correspondence with the class of star bodies.

If $G$ satisfies (a), (b) and

(c) $G(x+y) \leqq G(x)+G(y)$, then it necessarily satisfies (c). In this case the set $S$ is a convex body containing 0 . Throughout this paper it will be assumed that every convex body mentioned contains 0 . Since it is also true that, if $S$ is convex, its distance function satisfies $\left(c^{\prime}\right)$, the class of convex bodies is in one-to-one correspondence with the class of distance functions which satisfy $\left(c^{\prime}\right)$.

At times we shall have to consider a star body $S$ and its convex closure; the following representation theorem will be of service. 
THEOREM 1. Let $S$ be a star body with distance function $G, \bar{S}$ its convex closure. The distance function $F$ of $\bar{S}$ is representable in the form

$$
F(0)=0, \quad F(x)=\inf \left\{\alpha_{1} G\left(x_{1}\right)+\cdots+\alpha_{n} G\left(x_{n}\right)\right\} \quad \text { for } x \neq 0,
$$

where the infimum is to be taken over the elements $\alpha_{1}, \ldots, \alpha_{n}, x_{1}, \ldots, x_{n}$ such that

$$
\begin{gathered}
x=\alpha_{1} x_{1}+\cdots+\alpha_{n} x_{\imath}, \quad \alpha_{1} \geqq 0, \ldots, \alpha_{n} \geqq 0, \\
\alpha_{1}+\cdots+\alpha_{n}=1, \quad \operatorname{det}\left(x_{1}, \ldots, x_{n}\right) \neq 0 .
\end{gathered}
$$

For the choices of $\alpha_{1}, \ldots, \alpha_{n}$ allowed we have

$$
\alpha_{1} G\left(x_{1}\right)+\cdots+\alpha_{n} G\left(x_{n}\right) \geqq 0
$$

and so the infimum exists for each $x$. Let $F^{\prime}$ be the function defined by this infimum when $x \neq 0$ and take $F^{\prime}(0)=0$. We will show that $F^{\prime}(x)=F(x)$. Since this is true for $x=0$, we assume from here on that $x \neq 0$.

For $\lambda>0$,

$$
\alpha_{1} G\left(\lambda x_{1}\right)+\cdots+\alpha_{n} G\left(\lambda x_{n}\right)=\lambda\left[\alpha_{1} G\left(x_{1}\right)+\cdots+\alpha_{n} G\left(x_{n}\right)\right]
$$

from which it follows that

$$
F^{\prime}(\lambda x)=\lambda F^{\prime}(x)
$$

Suppose we have two representations of $x$, viz.

$$
x=\alpha_{1} x_{1}+\cdots+\alpha_{n} x_{n}=\alpha_{1}^{\prime} x_{1}^{\prime}+\cdots+\alpha_{n}^{\prime} x_{n}^{\prime},
$$

where $x_{j}^{\prime}=\lambda_{j} x_{j}, \lambda_{j}>0, j=1,2, \ldots, n$, and $\alpha_{1}^{\prime}, \ldots, \alpha_{n}^{\prime}$ satisfy the conditions (2.1). Since

$$
\left(\alpha_{1}-\alpha_{1}^{\prime} \lambda_{1}\right) x_{1}+\cdots+\left(\alpha_{n}-\alpha_{n}^{\prime} \lambda_{n}\right) x_{n}=0
$$

and $x_{1}, \ldots, x_{n}$ are linearly independent, $\alpha_{j}=\alpha_{j}^{\prime} \lambda_{j}$. Moreover, because $G$ satisfies (b),

$$
\alpha_{j}^{\prime} G\left(x_{j}^{\prime}\right)=\alpha_{j}^{\prime} G\left(\lambda_{j} x_{j}\right)=\alpha_{j}^{\prime} \lambda_{j} G\left(x_{j}\right)=\alpha_{j} G\left(x_{j}\right) ;
$$

therefore

$$
\alpha_{1} G\left(x_{1}\right)+\cdots+\alpha_{n} G\left(x_{n}\right)=\alpha_{1}^{\prime} G\left(x_{1}^{\prime}\right)+\cdots+\alpha_{n}^{\prime} G\left(x_{n}^{\prime}\right) .
$$

We shall now prove that if $x$ is in $\partial \bar{S}$, then $F^{\prime}(x)=1$. For such an $x$ lies in some support plane of $\bar{S}$; let

$$
x=\alpha_{1} x_{1}+\cdots+\alpha_{n} x_{n}
$$

be one representation of $x$ and let $x_{1}^{\prime}, \ldots, x_{n}^{\prime}$ be the intersection of the rays $R_{x_{1}}, \ldots, R_{x_{n}}$ with that support plane. From these points we obtain a second representation where $\alpha_{1}^{\prime}, \ldots, \alpha_{n}^{\prime}, x_{1}^{\prime}, \ldots, x_{n}^{\prime}$ satisfy the conditions $(2.1), x_{j}^{\prime}=\lambda_{j} x_{j}$, 
$\lambda_{j}>0$. The points $x_{1}^{\prime}, \ldots, x_{n}^{\prime}$ are either exterior to or boundary points of $S$ and so $G\left(x_{j}^{\prime}\right) \geqq 1$ from which it follows that

$$
\alpha_{1}^{\prime} G\left(x_{1}^{\prime}\right)+\cdots+\alpha_{n}^{\prime} G\left(x_{n}^{\prime}\right) \geqq 1 .
$$

With the aid of (2.3), we conclude that $F^{\prime}(x) \geqq 1$.

Since $x$ is in $\partial \bar{S}$, there is a representation of the type (2.4) in which $x_{1}, \ldots, x_{p}$ are in $\partial S$ and $\alpha_{p+1}=\cdots=\alpha_{n}=0$. With this choice we get $\alpha_{1} G\left(x_{1}\right)+\cdots+\alpha_{n} G\left(x_{n}\right)=1$ and so $F^{\prime}(x)=1$.

Denote by $z$ the point $R_{x} \cap \partial \bar{S}$; then $x=\|x\| z /\|z\|$ and

$$
F(x)=\|x\| /\|z\|=\|x\| F^{\prime}(z) /\|z\|=F^{\prime}(x)
$$

by (2.2) which completes the proof. Note that the infimum is actually an attained minimum.

The class of star bodies is partially ordered by inclusion; this induces a partial ordering in the set of distance functions. More precisely, if $S_{i}, i=0,1$, are star bodies with distance functions $G_{i}$, then $S_{0} \subseteq S_{1}$ and $G_{0}(x) \geqq G_{1}(x)$ over $R_{n}$ are equivalent assertions.

The convergence of a sequence $\left\{S_{j}\right\}$ of star bodies to a star body $S$ will be defined as follows. Let $G_{j}$ be the distance function of $S_{j}, G$ that of $S$. We say $\left\{S_{j}\right\}$ converges to $S$ if $\left\{G_{j}\right\}$ converges to $G$ uniformly over $\partial E$. We next prove a convergence theorem.

THEOREM 2. Suppose a sequence $\left\{S_{j}\right\}$ of star bodies satisfies

$$
b_{0} E \subseteq S_{j} \subseteq b_{1} E, \quad 0<b_{0} \leqq b_{1}, \quad j=1,2, \ldots,
$$

and that the corresponding sequence $\left\{G_{j}\right\}$ of distance functions converges uniformly over $\partial E$ to a function $G$. Then $\left\{S_{j}\right\}$ converges to a star body $S$ and $G$ is its distance function. Moreover the sequence $\left\{\bar{S}_{j}\right\}$ of convex closures converges to $\bar{S}$.

In the first part of the theorem we need only show that $G$ is a distance function. The distance functions of $b_{i} E$ are $\|x\| / b_{i}$, hence $0<1 / b_{1} \leqq G_{j}(x) \leqq 1 / b_{0}$ over $\partial E$ and this must be true for $G$. Further the positive homogeneity (b) of the functions $G_{j}$ entails that of $G$. Finally the uniform convergence of the continuous function $G_{j}$ implies the continuity of $G$ over $\partial E$. Clearly the homogeneity of $G$ implies its continuity for all $x$ and from $G_{j}(0)=0$ we have $G(0)=0$. Thus $G$ meets requirements (a), (b), (c) as was to be shown.

By Theorem 1, if $F_{j}$ is the distance function of $\bar{S}_{j}$ and $F$ that of $\bar{S}$,

$$
\begin{aligned}
F_{j}(x) & =\min \left[\alpha_{1} G_{j}\left(x_{1}\right)+\cdots+\alpha_{n} G_{j}\left(x_{n}\right)\right], \\
F(x) & =\min \left[\alpha_{1} G\left(x_{1}\right)+\cdots+\alpha_{n} G\left(x_{n}\right)\right]
\end{aligned}
$$

for $x \neq 0$ where the minima are taken over representations satisfying (2.1). The convergence assumption, viz. $G_{j} \rightarrow G$ over $\partial E$, can be written

$$
\left|G_{j}(x)-G(x)\right|<\varepsilon\|x\|, \text { for all } j>N(\varepsilon) \text { and for all } x,
$$


where $N(\varepsilon) \rightarrow \infty$ monotonically as $\varepsilon \rightarrow 0$. In (2.5) the minima are attained and so for each $j>N\left(b_{0} \varepsilon / b_{1}\right)$ we may determine $\alpha_{1}^{\prime}, \ldots, \alpha_{n}^{\prime}, x_{1}^{\prime}, \ldots, x_{n}^{\prime}$ satisfying (2.1) such that

$$
F_{j}(x)=\alpha_{1}^{\prime} G_{j}\left(x_{1}^{\prime}\right)+\cdots+\alpha_{n}^{\prime} G_{j}\left(x_{n}^{\prime}\right) ;
$$

further let $\alpha_{1}, \ldots, \alpha_{n}, x_{1}, \ldots, x_{n}$ satisfy (2.1) and be such that

$$
F(x)=\alpha_{1} G\left(x_{1}\right)+\cdots+\alpha_{n} G\left(x_{n}\right) .
$$

First, if $F(x) \geqq F_{j}(x)$, we have from the minimal character of $F$ :

$$
\begin{aligned}
0 & \leqq F(x)-F_{j}(x) \leqq \alpha_{1}^{\prime}\left(G\left(x_{1}^{\prime}\right)-G_{j}\left(x_{1}^{\prime}\right)\right)+\cdots+\alpha_{n}^{\prime}\left(G\left(x_{n}^{\prime}\right)-G_{j}\left(x_{n}^{\prime}\right)\right) \\
& \leqq \alpha_{1}^{\prime}\left|G\left(x_{1}^{\prime}\right)-G_{j}\left(x_{1}^{\prime}\right)\right|+\cdots+\alpha_{n}^{\prime}\left|G\left(x_{n}^{\prime}\right)-G_{j}\left(x_{n}^{\prime}\right)\right| \\
& \leqq\left(\varepsilon b_{0} / b_{1}\right)\left(\alpha_{1}^{\prime}\left\|x_{1}^{\prime}\right\|+\cdots+\alpha_{n}^{\prime}\left\|x_{n}^{\prime}\right\|\right)
\end{aligned}
$$

in virtue of (2.6). By similar reasoning, if $F_{j}(x) \geqq F(x)$, we deduce from the minimal character of $F_{j}$ and from (2.6) that

$$
0 \leqq F_{j}(x)-F(x) \leqq\left(\varepsilon b_{0} / b_{1}\right)\left(\alpha_{1}\left\|x_{1}\right\|+\cdots+\alpha_{n}\left\|x_{n}\right\|\right) .
$$

In the first case suppose $x$ is on $\partial \bar{S}_{j}$; then $x_{1}^{\prime}, \ldots, x_{n}^{\prime}$ can be chosen on $\partial S_{j}$ and from the bounds on $S_{j}$ we have $\left\|x_{1}^{\prime}\right\| \leqq b_{1}, \ldots,\left\|x_{n}^{\prime}\right\| \leqq b_{1}$ and so $\left|F(x)-F_{j}(x)\right|<\varepsilon b_{0}$. But then, dividing by $\|x\| \geqq b_{0}$, (since $\bar{S}_{j} \supseteq b_{0} E$ ), we obtain

$$
\left|F(u)-F_{j}(u)\right|<\varepsilon, \text { for } j>N\left(b_{0} \varepsilon / b_{1}\right) \text { and } u \text { on } \partial E \text {. }
$$

In the second case, suppose $x$ is on $\partial \bar{S}$; then $x_{1}, \ldots, x_{n}$ can be chosen on $\partial S$ and, in the same way as before but using the bounds on $S$, we have (2.7). Therefore $F_{j} \rightarrow F$ uniformly over $\partial E$ and so, by the first part of the theorem, since $b_{0} E \subseteq \bar{S}_{j}$ $\subseteq b_{1} E$, we conclude that $\left\{\bar{S}_{j}\right\}$ converges to $\bar{S}$.

Let $K$ be a convex body. In addition to its distance function $F$, one defines, as a second characterizing function for $K$, its support function $H$ over $R_{n}$ by $H(x)=\max _{z \in K}(x, z)$. For each $x \neq 0$, the half-space $h(x)$ of points $y$ such that $(y, x) \leqq H(x)$ contains $K$ and its bounding plane contains at least one point of $\partial K$. $h(x)$ is called a supporting half-space of $K$ and its boundary is the support plane of $K$ in the direction $x$. We have $K=\bigcap_{u \in \partial E} h(u)$.

Let $y$ be the intersection of $R_{x}$ with the support plane in the direction $x$. An alternative representation for $H$ is

$$
H(x)=\|x\|\|y\|, \text { for } x \neq 0, \quad H(0)=0 .
$$

If $z \neq 0$ and $u=z /\|z\|$, then $z / F(z)=u / F(u)$. Suppose $z$ is on $R_{z} \cap K$, then $F(z) \leqq 1$ and so for such $z(x, z) \leqq(x, u) / F(u)$ with equality if and only if $z$ is on $\partial K$. This gives yet another useful representation of $H$, viz.

$$
H(x)=\max _{u \in \partial E}(x, u) / F(u) .
$$

The support function of any convex body satisfies conditions (a), (b), (c'). And, conversely, any function meeting these requirements is the support function of a convex body. 
Let us suppose that $G$ is a function, defined over $R_{n}$, which satisfies (a), (b), (c'): viewed as a distance function, $G$ determines a convex body $K$; viewed as a support function, $G$ determines a second convex body $\hat{K}$. $K$ and $\hat{K}$ are polar reciprocal with respect to $E$. In more detail, let $x$ be any point of $K$ other than 0 ; with $x$ we associate the half-space $(y, x) \leqq 1 . \hat{K}$ is the intersection of these half-spaces. We may describe $K$ as the convex closure of its boundary points, i.e., $K=\bigcup x$, where the union is to be taken over $x$ for which $G(x)=1$. In forming $\hat{K}$ as the intersection of the half-spaces $(y, x) \leqq 1$, we may restrict ourselves to those half-spaces corresponding to $x$ on $\partial K$. Set $u=x /\|x\|$; then $G(u)=1 /\|x\|$ and so the half-space $(y, x) \leqq 1$ is the half-space $g(u):(y, u) \leqq G(u)$. We have

$$
(\bar{\bigcup} x)^{\wedge}=\bigcap_{u \in \partial E} g(u) .
$$

Relation (2.9) however remains true even if $G$ satisfies (a), (b), (c) and not (c').

We note that polar reciprocation is involutory, i.e., $(\hat{K})^{\wedge}=K$.

By a polar reciprocation argument and equation (2.8), we find as another representation of the distance function $F$ of a convex body $K$

$$
F(x)=\max _{u \in \partial E}(x, u) / H(u) .
$$

Also by this type of argument we can establish the following

Corollary to Theorem 1. Suppose $G$ is a function satisfying conditions (a), (b), (c) and suppose $K$ to be the convex body defined by $K=\bigcap_{u \in \partial E} g(u)$. The support function of $K$ is $H$ defined by

$$
H(x)=\inf \left\{\alpha_{1} G\left(x_{1}\right)+\cdots+\alpha_{n} G\left(x_{n}\right)\right\}, \text { for } x \neq 0,
$$

where $\alpha_{1}, \ldots, \alpha_{n}, x_{1}, \ldots, x_{n}$ satisfy $(2.1)$, and $H(0)=0$.

By (2.9) $\hat{K}=\bar{\bigcup} x$ where the union taken over all $x$ satisfying $G(x) \leqq 1$. Then $\hat{K}$ is the convex closure of a star body whose distance function is $G(x)$. In virtue of Theorem 1, the distance function of $\hat{K}$ is the $H$ described in the statement of the corollary. Therefore this $H$ is the support function of $K$.

The partial ordering of convex bodies by inclusion induces a partial ordering among support functions. Thus $K_{0} \subseteq K_{1}$ and $H_{0}(x) \leqq H_{1}(x)$ over $R_{n}$ are equivalent assertions where $H_{i}$ is the support function of $K_{i}$. It follows that $\hat{K}_{0} \supseteq \hat{K}_{1}$ is equivalent to $K_{0} \subseteq K_{1}$.

The deviation $\delta$ defined by

$$
\delta\left(K_{0}, K_{1}\right)=\max _{v \in \partial E}\left|H_{0}(v)-H_{1}(v)\right|
$$

is a metric for the space of convex bodies. This provides a second definition of convergence which is equivalent to the earlier one applied to the space of convex 
bodies. First note that if $F_{j} \rightarrow F$ over $\partial E$, where $F_{j}, F$ are the distance functions of $K_{j}, K$, then there are positive numbers $b_{0} \leqq b_{1}$ such that

$$
b_{0} E \subseteq K_{j} \subseteq b_{1} E, \quad b_{0} E \subseteq K \subseteq b_{1} E,
$$

that is, over $\partial E$

$$
b_{0} \geqq F_{j}(u) \geqq b_{1}, \quad b_{0} \geqq F(u) \geqq b_{1} .
$$

Hence, by (2.8),

$$
\begin{aligned}
\left|H_{j}(v)-H(v)\right| & =\left|\max _{u \in \partial E} \frac{(v, u)}{F_{j}(u)}-\max _{u \in \partial E} \frac{(v, u)}{F(u)}\right| \\
& \leqq \max _{u \in \partial E}|(v, u)|\left|F_{j}(u)-F(u)\right| / F_{j}(u) F(u) \\
& \leqq \max _{u \in \partial E E}\left|F_{j}(u)-F(u)\right| / b_{1}^{2},
\end{aligned}
$$

from which it follows that $H_{j} \rightarrow H$ uniformly over $\partial E$. Similarly, with the aid of (2.10), we complete the equivalence proof.

The items of background which have been mentioned in this section without proof can be found in [1] and [10].

3. Suppose $F$ is a real-valued function over $R_{n} \times T$ and that $F$ satisfies these three conditions:

(A) For each $x$ in $\partial E, F(x, t)$ is measurable over $T$ and $b_{0} \leqq F(x, t) \leqq b_{1}$ for some pair of positive numbers $b_{0}, b_{1}$ independent of $x$ in $\partial E$;

(B) For each $t$ in $T$ and each $\lambda>0, F(\lambda x, t)=\lambda F(x, t)$;

(C) For each $t$ in $T, F(x, t)$ is continuous over $R_{n}$.

These conditions imply that $F$ satisfies (a), (b), (c) for each $t$; since (B), (C) are conditions (b), (c), only (a) must be shown. By (B) if $x \neq 0$,

$$
0<b_{0}\|x\| \leqq F(x, t) \leqq b_{1}\|x\| .
$$

Hence $F(x, t) \rightarrow 0$ as $x \rightarrow 0$ and so, by (C), $F(0, t)=0$. This proves (a). In view of this, with each $t$ in $T$ we may associate a unique star body $S(t)$ in $R_{n}$ which has $F(x, t)$ as its distance function. Such a mapping of $T$ into the space of star bodies will be called a bounded, measurable family $\mathbf{S}$ of star bodies over $T$. The boundedness means, geometrically, that

$$
\left(1 / b_{1}\right) E \subseteq S(t) \subseteq\left(1 / b_{0}\right) E
$$

for all $t$ and is a consequence of (A).

Our interest will centre on those cases in which the star bodies $S(t)=K(t)$ are convex, i.e., when $F$ satisfies

(C') $F(x+y, t) \leqq F(x, t)+F(y, t)$ for each $t$ in $T$, in which case (C) is a consequence of $(B)$ and $\left(C^{\prime}\right)$. Under these circumstances we shall call $K$ a bounded, measurable family of convex bodies over $T$. Let $H(x, t)$ be the family of support functions associated with $\mathbf{K}$. 
THEOREM 3. In order that $\mathbf{K}$ be a bounded, measurable family of convex bodies, it is necessary and sufficient that the associated support function $H(x, t)$ satisfy conditions (A), (B), ( $\left.\mathrm{C}^{\prime}\right)$.

Condition (A) is the only one in question; we must show that $H(x, t)$ is measurable over $T$ for each $x$ if and only if this is true of $F(x, t)$. Assume $F$ satisfies (A); let $x$ be fixed and define over $\partial E \times T$ :

$$
\psi(v, t)=(v, x) / F(v, t) .
$$

$\psi$ is bounded and measurable over $T$ for each $v$ in $\partial E$ and is continuous in $v$ over $\partial E$ for each $t$. Select a denumerable subset $V$ of $\partial E$ which is everywhere dense. From the representation (2.8) of $H$ and the continuity of $\psi$ in $v$, we have

$$
H(x, t)=\sup _{v \in V} \psi(v, t) .
$$

Such an upper envelope is necessarily measurable, cf. [8, p. 84]. The boundedness of $H(v, t)$ follows from (3.1), the induced partial ordering of support functions, and the fact that $r E$ has $r\|x\|$ as its support function. Hence, if $\mathbf{K}$ is a bounded, measurable family, $H(x, t)$ satisfies $(\mathrm{A}),(\mathrm{B}),\left(\mathrm{C}^{\prime}\right)$.

Suppose $H(x, t)$ satisfies (A), (B), ( $\left.\mathrm{C}^{\prime}\right)$. Let us consider the polar reciprocal family $\hat{\mathbf{K}}$ of convex bodies $\hat{K}(t) . H(x, t)$ is the associated distance function for $\hat{\mathbf{K}}$ and so $\hat{\mathbf{K}}$ is a bounded, measurable family. Consequently, by our preceding discussion, the associated support function $F(x, t)$ of $\hat{\mathbf{K}}$ satisfies $(\mathrm{A}),(\mathrm{B}),\left(\mathrm{C}^{\prime}\right)$. This completes the theorem, and we have the following corollary.

Corollary to TheOREM 3. If either one of the families $\mathbf{K}, \hat{\mathbf{K}}$ is bounded and measurable, so is the other.

From a family $\mathbf{S}$ of star bodies we can construct a family $\mathbf{K}$ of convex bodies by taking convex closures, that is $\bar{S}(t)=K(t)$. The next theorem relates the boundedness and measurability of $\mathbf{S}$ and $\mathbf{K}$.

THEOREM 4. If $\mathbf{S}$ is a bounded, measurable family of star bodies over $T$, then the family $\mathbf{K}$ of convex closures is a bounded, measurable family of convex bodies over $T$.

From $b_{0} E \subseteq S(t) \subseteq b_{1} E$ over $T$ we have $b_{0} E \subseteq \bar{S}(t) \subseteq b_{1} E$ and so $\mathbf{K}$ is bounded.

Let $G(x, t)$ be the distance function of $S(t)$. $G$ satisfies (A), (B), (C). Denote by $F(x, t)$ the distance function of $\bar{S}(t)=K(t)$. We must show that $F$ satisfies (A), (B), $\left(C^{\prime}\right)$; the only point in question, however, is the first part of condition (A): we must show that $F(x, t)$ is measurable over $T$ for each $x$. Since $F(0, t)=0$ for all $t$, we may suppose $x \neq 0$.

Let $x$ be fixed and let $\Sigma$ be the space of elements $e=\left(\alpha_{1}, \ldots, \alpha_{n}, x_{1}, \ldots, x_{n}\right)$ where $\alpha_{1}, \ldots, \alpha_{n}, x_{1}, \ldots, x_{n}$ satisfy (2.1). Take as a metric in $\Sigma$

$$
d\left(e, e^{\prime}\right)=\left|\alpha_{1}-\alpha_{1}^{\prime}\right|+\cdots+\left|\alpha_{n}-\alpha_{n}^{\prime}\right|+\left\|x_{1}-x_{1}^{\prime}\right\|+\cdots+\left\|x_{n}-x_{n}^{\prime}\right\| .
$$

The function

$$
g(e, t)=\alpha_{1} G\left(x_{1}, t\right)+\cdots+\alpha_{n} G\left(x_{n}, t\right)
$$


is continuous over $\Sigma$; let $\Sigma^{\prime}$ be a denumerable subset of $\Sigma$ which is everywhere dense in $\Sigma$. By Theorem 1, and this continuity of $g, F(x, t)=\inf _{e \in \Sigma^{\prime}} g(e, t)$. Since $g(e, t)$ is measurable over $T$ for each $e, F(x, t)$ as a lower envelope is measurable over $T$ for each $x$.

Suppose $\mathbf{S}$ is a bounded, measurable family of star bodies; the distance function $F$ which is associated with $\mathbf{S}$ satisfies (A) over $T$ for each $x \neq 0$ and, therefore, so do the functions $F^{p}, \ln F$ where $0<|p| \leqq \infty$. Consequently, the means

$$
M_{p}^{t}(F(x, t))=M_{p}(F)
$$

exist for each $x \neq 0$ in $R_{n}$ and all $p$. The conventions adopted in the definition (1.1) of these means take care of the case $x=0$.

From their definitions we see directly that the functions $M_{p}(F)$ satisfy conditions (a) and (b). They also satisfy (c). Suppose $p \neq 0$ or $\pm \infty$ and that $\left\{x_{j}\right\}$ is a sequence of points of $R_{n}$ with limit $x \neq 0$. There are numbers $r, R$ such that $0<r \leqq\left\|x_{j}\right\| \leqq R$ for all but a finite number of indices. Set $f_{j}(t)=F^{p}\left(x_{j}, t\right)$; then

$$
0<f_{j}(t) \leqq\left(R b_{1}\right)^{p} \quad \text { for } p>0, \quad 0<f_{j}(t) \leqq\left(r b_{0}\right)^{p} \text { for } p<0 .
$$

By Lebesgue's convergence theorem and condition (C)

$$
\lim _{j \rightarrow \infty} \int_{T} f_{j}(t) d t=\int_{T} F^{p}(x, t) d t
$$

and the continuity of $z^{1 / p}$ in $z$ completes the demonstration of (c) for these cases.

If $\left\{x_{j}\right\}$ tends to 0 , we have for $\|x\| \neq$,0 ,

$$
0 \leqq M_{p}^{t}\left(F\left(x_{j}, t\right)\right)=\left\|x_{j}\right\| M_{p}^{t}\left(F\left(x_{j} /\left\|x_{j}\right\|, t\right)\right) \leqq b_{1}\left\|x_{j}\right\|,
$$

these estimates are also true for $\left\|x_{j}\right\|=0$ and so, in this instance,

$$
\lim _{j \rightarrow \infty} M_{p}^{t}\left(F\left(x_{j}, t\right)\right)=M_{p}^{t}(F(0, t))=0 .
$$

Thus $M_{p}(F)$ is continuous for all $x$ when $0<|p|<\infty$. The special cases $p=0$ or $\pm \infty$ can be treated similarly.

Consequently with each bounded, measurable family $\mathbf{S}$ of star bodies over $\boldsymbol{T}$ and each $p$ we may asscciate a $p$ th order mean star body $m_{p}^{t}(S(t))=m_{p}(\mathbf{S})$, namely one whose distance function is $M_{-p}(F)$.

When $p \geqq 1$, the function $M_{p}(F)$ satisfies (a), (b), (c') if we suppose $F$ satisfies (A), (B), $\left(C^{\prime}\right)$. Condition $\left(C^{\prime}\right)$ is the only one in question and, by Minkowski's inequality and $(B),\left(C^{\prime}\right)$ :

$$
\begin{aligned}
M_{p}^{t}\left(F\left((1-\vartheta) x_{0}+\vartheta x_{1}, t\right)\right) & \leqq M_{p}^{t}\left((1-\vartheta) F\left(x_{0}, t\right)+\vartheta F\left(x_{1}, t\right)\right) \\
& \leqq(1-\vartheta) M_{p}^{t}\left(F\left(x_{0}, t\right)\right)+\vartheta M_{p}^{t}\left(F\left(x_{1}, t\right)\right) \\
& =M_{p}^{t}\left(F\left((1-\vartheta) x_{0}, t\right)\right)+M_{p}^{t}\left(F\left(\vartheta x_{1}, t\right)\right)
\end{aligned}
$$

for $0 \leqq \vartheta \leqq 1$. 
We set $x=(1-\vartheta) x_{0}, y=\vartheta x_{1}$ and obtain

$$
M_{p}^{t}(F(x+y, t)) \leqq M_{p}^{t}(F(x, t))+M_{p}^{t}(F(y, t)) .
$$

Therefore, whenever $\mathbf{K}$ is a bounded, measurable family of convex bodies, the sets $m_{p}(\mathbf{K})$ are convex bodies for $p \leqq-1$. In general this will not be true for $p>-1$. For the family $\mathbf{K}$ we define a collection of means $\dot{M}_{p}(\mathbf{K})$, which are convex bodies for all $p$, by taking $\dot{M}_{p}(\mathbf{K})=\bar{m}_{p}(\mathbf{K})$. For the rest of the paper, unless the contrary is stated, we shall suppose $-\infty<p<\infty$. In terms of the distance function $F(x, t)$, the set $\dot{M}_{p}(\mathbf{K})$ is the convex closure of the set of points $x$ which satisfy

$$
M_{-p}^{t}(F(x, t)) \leqq 1 .
$$

A second family of means will be introduced next. Again we suppose $\mathbf{K}$ to be bounded and measurable. By the corollary to Theorem 3 we know that $\hat{\mathbf{K}}$ is also a bounded, measurable family and so we may form the means $\dot{M}_{-p}(\hat{\mathbf{K}})$ for each $p$. We define our second set of means $M_{p}(\mathbf{K})$ by

$$
M_{p}^{t}(K(t))=M_{p}(\mathbf{K})=\left[\dot{M}_{-p}(\hat{\mathbf{K}})\right]^{\wedge} .
$$

If we replace $\mathbf{K}$ by $\hat{\mathbf{K}}$ in (3.3) and take polar reciprocals of each side, we obtain

$$
\dot{M}_{-p}(\mathbf{K})=\hat{M}_{p}(\hat{\mathbf{K}}),
$$

in virtue of the involutory character of polar reciprocation.

There is an alternate and more direct representation of $M_{p}(\mathbf{K})$ in terms of the associated support function $H(x, t)$. By Theorem 3, $H$ satisfies (A), (B), (C') and so we may form the means $M_{p}(H)$. These means satisfy (a), (b), (c); hence $M_{p}(H)$ is the distance function of $m_{-p}(\hat{\mathbf{K}})$. The convex closure $\dot{M}_{p}(\hat{\mathbf{K}})$ is $\bigcup x$ where the union need only be taken over all $x$ for which $M_{p}^{t}(H(x, t))=1$.

By (2.9) and (3.3)

$$
(\bar{\bigcup} x)^{\wedge}=\bigcap_{u \in \partial E} h(u)=M_{p}(\mathbf{K}),
$$

where $h(u)$ is the half-space $(x, u) \leqq M_{p}^{t}(H(u, t))$.

In general $M_{p}(H)$ is not the support function of $M_{p}(\mathbf{K})$; it is, however, if $p \geqq 1$.

4. Some specialized cases of the means introduced in the preceding section will be taken up next.

The means $M_{p}$ and $\dot{M}_{p}$ of convex bodies in $R_{1}$ are particularly simple. A convex body $K$ in $R_{1}$ is a closed interval; we require $K$ to contain the origin and so $K$ is a set of points $x$ which satisfy $-a \leqq x \leqq b$, where $a>0, b>0$. The distance and support functions of $K$ are $F$ and $H$ defined by

$$
\begin{array}{lll}
F(x)=|x| / b, & H(x)=|x| b, & \text { when } x \geqq 0, \\
F(x)=|x| / a, & H(x)=|x| a, & \text { when } x \leqq 0 .
\end{array}
$$

From this we see that if $\mathbf{K}$ is a family of convex bodies $K(t):-a(t) \leqq x \leqq b(t)$, 
$t$ in $T$, then, in order that $\mathbf{K}$ be a bounded, measurable family, it is necessary and sufficient that the functions $a, b$ be measurable over $T$ and satisfy

$$
0<r \leqq a(t) \leqq R, \quad 0<r \leqq b(t) \leqq R
$$

for some constants $r, R$. Further, if $\mathbf{K}$ is a bounded, measurable family, the means $M_{p}(\mathbf{K})$ and $\dot{M}_{p}(\mathbf{K})$ coincide; indeed they are the interval $-M_{p}^{t}(a(t)) \leqq x \leqq M_{p}^{t}(b(t))$, since $M_{-p}^{t}(|x| / a(t))=|x| / M_{p}^{t}(a(t))$, and similarly for $b$.

The next special instance is that of elementary means. We call a family $\mathbf{K}$ of convex bodies in $R_{n}$ piecewise constant over $T$ if there is both a decomposition of $T$ into a finite number of disjoint, measurable subsets $\tau_{0}, \tau_{1}, \ldots, \tau_{r}$ and a collection of convex bodies $K_{0}, K_{1}, \ldots, K_{r}$ such that $K(t)=K_{j}$ for all $t$ in $\tau_{j}, j=0,1, \ldots, r$. Clearly such families are bounded and measurable. Let $\lambda_{j}$ be the measure of $\tau_{j}$; then $\lambda_{0}+\lambda_{1}+\cdots+\lambda_{r}=1$ and

$$
M_{p}^{t}(H(x, t))=\left[\lambda_{0} H_{0}^{p}(x)+\lambda_{1} H_{1}^{p}(x)+\cdots+\lambda_{r} H_{r}^{p}(x)\right]^{1 / p}
$$

when $p \neq 0$. Here $H_{j}$ is the support function of $K_{j}$. If $p=0$, we have

$$
M_{0}^{t}(H(x, t))=H_{0}^{\lambda^{o}}(x) H_{1}^{\lambda_{1}}(x) \cdots H_{r}^{\lambda_{r}}(x) .
$$

For piecewise constant families, (which without loss of generality will be assumed defined over $T_{1}$ ), we write

$$
M_{p}(\mathbf{K})=M_{p}\left(K_{0}, K_{1}, \ldots, K_{r} ; \lambda_{0}, \lambda_{1}, \ldots, \lambda_{r}\right) .
$$

These are the elementary means of finite collections of convex bodies. If $r=1$, we set $\lambda_{1}=\vartheta$ and $\lambda_{0}=1-\vartheta$ and write $M_{p}(\mathbf{K})=M_{p}\left(K_{0}, K_{1} ; \vartheta\right)$.

Similarly, for general $r$, we write

$$
\dot{M}_{p}(\mathbf{K})=\dot{M}_{p}\left(K_{0}, K_{1}, \ldots, K_{r} ; \lambda_{0}, \lambda_{1}, \ldots, \lambda_{r}\right)
$$

and, for $r=1, \dot{M}_{p}(\mathbf{K})=\dot{M}_{p}\left(K_{0}, K_{1} ; \vartheta\right)$.

The elementary means $M_{p}$ are discussed for $p \geqq 1$ in [4]; the elementary means $\dot{M}_{p}$ for $p \leqq-1$ are discussed in [5]. In the latter paper, the index $p$ equals the index $-p$ of the present paper.

The elementary mean $M_{1}\left(K_{0}, K_{1}, \ldots, K_{r} ; \lambda_{0}, \lambda_{1}, \ldots, \lambda_{r}\right)$ is of classical importance being the weighted vector or Minkowski sum $\lambda_{0} K_{0}+\lambda_{1} K_{1}+\cdots+\lambda_{r} K_{r}$ which is the set of points $x$ of the form $\lambda_{0} x_{0}+\lambda_{1} x_{1}+\cdots+\lambda_{r} x_{r}$ where $x_{j}$ is in $K_{j}$. This follows from the fact that the Minkowski sum so defined has the support function $\lambda_{0} H_{0}+\lambda_{1} H_{1}+\cdots+\lambda_{r} H_{r}$, cf. [1, p. 29].

More generally the mean $M_{1}(\mathbf{K})$ is connected with the Riemann-Minkowski integral of A. Dinghas [2], [3]. Here we suppose $\mathbf{K}$ to be a bounded family of convex bodies in $R_{n}$ with domain $T_{1}$. Let $\tau$ be any closed subinterval which contains $t$ and let $|\tau|$ be its length. We define the limit oscillation of $\mathbf{K}$ at $t$ to be

$$
\omega(t)=\lim _{|\tau| \rightarrow 0}\left[\sup _{t^{\prime}, t^{\prime \prime} \in \tau} \delta\left(K\left(t^{\prime}\right), K\left(t^{\prime \prime}\right)\right)\right]
$$


Let $\Pi$ be a decomposition of $T_{1}$ into disjoint intervals $\tau_{0}, \tau_{1}, \ldots, \tau_{r}$; let $\lambda_{j}=\left|\tau_{j}\right|$ and $t_{j}$ be some point in $\tau_{j}$. Finally set $\|\Pi\|=\max \left\{\lambda_{j}\right\}$. If

$$
\lim _{\|, \Pi\| \rightarrow 0}\left[\lambda_{0} K\left(t_{0}\right)+\lambda_{1} K\left(t_{1}\right)+\cdots+\lambda_{r} K\left(t_{r}\right)\right]
$$

exists, then, following Dinghas, this limit is the Riemann-Minkowski integral of $\mathbf{K}$ over $T_{1}$ and is denoted by

$$
J(\mathbf{K})=\int_{0}^{1} d t K(t)
$$

The preceding definition can have meaning if $\mathbf{K}$ is a family of closed, bounded and nonempty sets in $R_{n}$, providing we replace each $K\left(t_{j}\right)$ by its convex hull or, what comes to the same thing here, its convex closure. This is Dinghas' procedure for such $\mathbf{K}$. We restrict our considerations to families of convex bodies, however.

Dinghas proved that if the set $\Omega(\mathbf{K})$ of $t$-values at which $\omega(t)>0$ has zero measure, then $J(\mathbf{K})$ exists. We shall prove

THEOREM 5. If $\mathbf{K}$ is a bounded family of convex bodies over the interval $T_{1}$ and if $\Omega(\mathbf{K})$ is of zero measure, then the means $M_{p}(\mathbf{K})$ and $\dot{M}_{p}(\mathbf{K})$ exist for all real $p$ and

$$
M_{1}(\mathbf{K})=\int_{0}^{1} d t K(t) .
$$

From the definition of the deviation $\delta$ we see that the vanishing of the limit oscillation is equivalent to

$$
\lim _{|\tau| \rightarrow 0}\left[\sup _{t^{\prime}, t^{\prime \prime} \in \tau}\left(\max _{u \in \partial E}\left|H\left(u, t^{\prime}\right)-H\left(u, t^{\prime \prime}\right)\right|\right)\right]=0
$$

or

$$
\lim _{|\tau| \rightarrow 0}\left[\sup _{t^{\prime}, t^{\prime \prime} \in \tau}\left|H\left(x, t^{\prime}\right)-H\left(x, t^{\prime \prime}\right)\right|\right]=0
$$

for each $x$ in $R_{n}$. This is to say that, for each $x, H(x, t)$ is continuous at $t$ if and only if $\omega(t)=0$. Hence the condition that $\Omega(\mathbf{K})$ be of zero measure amounts to this: the set of $t$-values at which $H(x, t)$ is discontinuous is of zero measure. By assumption $H(x, t)$ is bounded in $t$ for each $x$. These conditions insure the Riemann integrability of $H(x, t)$ over $T_{1}$ and so suffice for its measurability. From Theorem 3 it follows that $\mathbf{K}$ is a bounded and measurable family; this establishes the first conclusion.

To demonstrate the second conclusion, we assume $J(\mathbf{K})$ and $M_{1}(\mathbf{K})$ exist, the former being the limit of approximating sums $M_{1}\left(K_{0}, K_{1}, \ldots, K_{r} ; \lambda_{0}, \lambda_{1}, \ldots, \lambda_{r}\right)$. These last convex bodies have support functions

$$
\lambda_{0} H\left(x, t_{0}\right)+\lambda_{1} H\left(x, t_{1}\right)+\cdots+\lambda_{r} H\left(x, t_{r}\right)
$$

which tend to $M_{1}(H)$ because $H(x, t)$ is Riemann integrable over $T_{1}$. Since $M_{1}(H)$ is the support function of $M_{1}(\mathbf{K}), J(\mathbf{K})=M_{1}(\mathbf{K})$. 
In what follows the domain $T$ is again general.

Let $\rho \cdot K$ signify the image of set $K$ under a rotation $\rho$ from $\mathscr{R}_{n}$; we denote by $\rho \cdot \mathbf{K}$ the family of images of members of $\mathbf{K}$ under $\rho$. If $G$ is the support or distance function of $K$ and $G^{\prime}$ the corresponding function for $\rho \cdot K$, then $G^{\prime}(x)=G\left(\rho^{-1} \cdot x\right)$ for all $x$ in $R_{n}$. From this it follows immediately that the means $M_{p}$ and $\dot{M}_{p}$ are isotropic, by which we mean that if $M$ is any one of these means, then

$$
\rho \cdot M(\mathbf{K})=M(\rho \cdot \mathbf{K}) .
$$

We next consider sections by and projections onto a linear subspace. Suppose $L\left(u_{1}, u_{2}, \ldots, u_{r}\right)=L$ is the orthogonal complement of the space spanned by the orthonormal vectors $u_{1}, u_{2}, \ldots, u_{r}, 1 \leqq r \leqq n-1$. $K / L$ will mean the orthogonal projection of $K$ onto $L$. The families $\mathbf{K}^{\star}$ and $\mathbf{K}^{*}$ defined by

$$
K^{\star}(t)=K(t) \cap L, \quad \text { and } \quad K^{*}(t)=K(t) / L
$$

are to be thought of as families of convex bodies in $L$. The distance function of $\mathbf{K}^{\star}$ is the restriction of $F(x, t)$ to points $x$ in $L$, and the support function of $\mathbf{K}^{*}$ is the restriction of $H(x, t)$ to such points. Therefore if $\mathbf{K}$ is a bounded and measurable family in $R_{n}, \mathbf{K}^{\star}$ and $\mathbf{K}^{*}$ are bounded and measurable families in $L$. Consequently, the existence of the means of $\mathbf{K}$ entails the existence of the means of $\mathbf{K}^{\star}$ and $\mathbf{K}^{*}$ in $L$.

Suppose $K$ is a fixed convex body and $F(x)$ its distance function. We form the family $\mathbf{K}$ defined over the rotations $\rho$ of $\mathscr{R}_{n}$ by $K(\rho)=\rho \cdot K$. Note that the distance function of $K(\rho)$ is $F(x, \rho)=F\left(\rho^{-1} \cdot x\right)$. We next form $\dot{M}_{p}(\mathbf{K})$; this is the convex closure of the star body whose distance function is $M_{-p}^{\rho}\left(F\left(\rho^{-1} \cdot x\right)\right)$. But the star body in question is a sphere because, if $\rho_{0}$ is any rotation

$$
M_{-p}^{\rho}\left(F\left(\rho^{-1} \rho_{0} \cdot x\right)\right)=M_{-p}^{\rho}\left(F\left(\rho^{-1} \cdot x\right)\right),
$$

i.e., the star body is invariant under all rotations. Hence $M_{-p}^{\rho}\left(F\left(\rho^{-1} \cdot x\right)\right)$ is the distance function of the sphere $\dot{M}_{p}(\mathbf{K})$; let $\dot{r}_{p}$ be the radius of the sphere. We have

$$
\dot{r}_{p}=1 / M_{-p}^{\rho}\left(F\left(\rho^{-1} \cdot u_{1}\right)\right)
$$

where $u_{1}$ is any fixed unit vector.

This radius can be described more simply. Consider an orthonormal system of $n-1$ vectors, the first of which is $u_{1}$, say $u_{1}, u_{2}, \ldots, u_{n-1}$. There is a one-to-one correspondence between the rotations $\rho^{-1}$ and the orthonormal systems $\rho^{-1} \cdot u_{1}$, $\rho^{-1} \cdot u_{2}, \ldots, \rho^{-1} \cdot u_{n}$. Moreover, with each of these latter systems we may associate a point $v=\left(v_{1}, v_{2}, \ldots, v_{n-1}\right)$ in $\partial E_{n} \times \partial E_{n-1} \times \cdots \times \partial E_{2}$ where $E_{k}$ is the unit sphere in $R_{k}$ centered at the origin. We effect this by taking the end point of $\rho^{-1} \cdot u_{1}$ to be the point $v_{1}$ in $\partial E_{n}$; then $\rho^{-1} \cdot u_{2}$ is in the orthogonal complement of $v_{1}$, hence its endpoint $v_{2}$ lies in $\partial E_{n-1}$ and so on. In this way we establish a bicontinuous, oneto-one correspondence between the rotations $\rho^{-1}$ and the points of $\partial E_{n} \times \partial E_{n-1}$ $\times \cdots \times \partial E_{2}$, cf. [10, pp. 226-227]. We set $d \omega=d \omega_{n} d \omega_{n-1} \cdots d \omega_{2}$ where $d \omega_{k}$ is the 
differential of surface area of $\partial E_{k}$ and $\omega_{k}$ is its total area. Write $F\left(v_{1}\right)=1 / f\left(v_{1}\right)$; $f\left(v_{1}\right)$ is the distance from the origin to $\partial K$ in the direction $v_{1}$. Then

$M_{-p}^{\rho}\left(F\left(\rho^{-1} \cdot u_{1}\right)\right)=\left[\frac{1}{\omega_{n} \omega_{n-1} \cdots \omega_{2}} \int_{\partial E_{n}} \int_{\partial E_{n-1}} \cdots \int_{\partial E_{2}} f^{p}\left(v_{1}\right) d \omega_{n} d \omega_{n-1} \cdots d \omega_{2}\right]^{-1 / p}$, or $\dot{r}_{p}=\sqrt[p]{ }\left[\int_{\partial E_{n}} f^{p}\left(v_{1}\right) d \omega_{n} / \omega_{n}\right]$. In particular

$$
\dot{r}_{n}=\sqrt[n]{\left[n V(K) / \omega_{n}\right]}
$$

where $V(K)$ is the volume of $K ; \dot{M}_{n}^{o}(\rho \cdot K)$ is a sphere of the same volume as $K$.

The quantities $\dot{r}$ are rotation invariants of $K$; we write $\dot{r}_{p}(K)$.

A corresponding collection of set functions $r_{p}(K)$ can be introduced with the aid of the means $M_{p}$. We form $M_{p}(\mathbf{K})$; note that $M_{p}(K)=M_{p}\left(\rho_{0} \cdot K\right)=\rho_{0} \cdot M_{p}(K)$ for each $\rho_{0}$ in $\mathscr{R}_{n}$ and so conclude that $M_{p}(\mathbf{K})$ is a sphere whose radius we denote by $r_{p}$. We have

$$
r_{p}=M_{p}^{\rho}\left(H\left(\rho^{-1} \cdot u_{1}\right)\right)=\sqrt[p]{p}\left[\int_{\partial E_{n}} h^{p}\left(v_{1}\right) d \omega_{n} / \omega_{n}\right]
$$

as before, where $H(x)$ is the support function of $K$ and $h\left(v_{1}\right)$ the distance from the origin to the support plane of $K$ in the direction of $v_{1}$. In particular $r_{1}(K)=B(K) / 2$ where $B(K)$ is the mean width of $K ; M_{1}^{\rho}(\rho \cdot K)$ is a sphere of the same mean width as $K$.

The set functions $V(K)$ and $\omega_{n} B(K) / 2 n$ are two particular mean cross-sectional measures (Quermassintegral). More generally, if we consider Steiner's polynomial expression for $V\left(K+\lambda E_{n}\right)$, viz.

$$
V\left(K+\lambda E_{n}\right)=\sum_{p=0}^{n}\left(\begin{array}{l}
n \\
p
\end{array}\right) W_{p}(K) \lambda^{p},
$$

then the coefficient $W_{p}(K)$ is called the $p$ th mean cross-sectional measure of $K$. In particular, if we set $\kappa_{n}=\omega_{n} / n$, then

$$
W_{0}(K)=V(K), \quad W_{n-1}(K)=\kappa_{n} B(K) / 2, \quad W_{n}(K)=\kappa_{n} .
$$

Thus we may write

$$
\dot{M}_{n}^{\rho}(\rho \cdot K)=\sqrt[n]{ }\left(W_{0}(K) / \kappa_{n}\right) E_{n}, \quad M_{1}^{\rho}(\rho \cdot K)=\left(W_{n-1}(K) / \kappa_{n}\right) E_{n} .
$$

The first of these formulae will be generalized.

Let $u_{1}, \ldots, u_{n-1}$ be a fixed orthonormal system of vectors and $\rho$ a rotation in $\mathscr{R}_{n}$; we write $v_{i}$ for $\rho^{-1} \cdot u_{i}, i=1, \ldots, n-1$. If $L_{n-p}$ is the linear subspace orthogonal to $u_{1}, \ldots, u_{p}$, then $\rho \cdot L_{n-p}$ is orthogonal to $v_{1}, \ldots, v_{p}$. The convex bodies $K / \rho \cdot L_{n-p}$ make up a family in $R_{n-p}$ defined over $\mathscr{R}_{n}$. Equally well, we may take the domain of the family to be the set $\partial E_{n} \times \partial E_{n-1} \times \cdots \times \partial E_{2}$ in virtue of our earlier remarks. Clearly the family is bounded and measurable. 
For the moment write $V\left(v_{1}, \ldots, v_{p}\right)$ for $V\left(K / \rho \cdot L_{n-p}\right)$. By repeated applications of Kubota's formulae, cf. [1, p. 49] and [6], we obtain

$$
\begin{aligned}
& W_{p}(K)=\frac{\gamma}{\omega_{n} \cdots \omega_{n-p+1} \kappa_{n-p}} \int_{\partial E_{n}} \cdots \int_{\partial E_{n-p+1}} V\left(v_{1}, \ldots, v_{p}\right) d \omega_{n}\left(v_{1}\right) \cdots d \omega_{n-p+1}\left(v_{p}\right), \\
& p=1,2, \ldots, n-1 \text {, }
\end{aligned}
$$

where $d \omega_{n-k}\left(v_{k+1}\right)$ indicates that that integration is with respect to $v_{k+1}$ over the spherical surface $\partial E_{n-k}$ of dimension $n-k-1$, and $\gamma$ is a constant independent of $K$. Since $V\left(v_{1}, \ldots, v_{p}\right)$ does not depend on $v_{p+1}, \ldots, v_{n}$ we may carry out further integrations and represent $W_{p}(K) / \gamma$ as

$$
\frac{1}{\omega_{n} \cdots \omega_{2}} \int_{\partial E_{n}} \cdots \int_{\partial E_{2}} \frac{V\left(v_{1}, \ldots, v_{p}\right)}{\kappa_{n-p}} d \omega_{n}\left(v_{1}\right) \cdots d \omega_{2}\left(v_{n-1}\right) .
$$

These integrations can be viewed as an integration over the rotation group $\mathscr{R}_{n}$. We use next (4.2) and obtain

$$
\left[W_{p}(K) / \gamma\right]^{1 /(n-p)}=M_{n-p}^{\rho}\left(\dot{r}_{n-p}\left(K / \rho \cdot L_{n-p}\right)\right) .
$$

Consider the sphere $\left[W_{p}(K) / \gamma\right]^{1 /(n-p)} E_{n-p}$; from the preceding we see that if, over $\mathscr{R}_{n}$, we define the family $\mathbf{C}$ of spheres in $R_{n-p}$ by $C(\rho)=\dot{r}_{n-p}\left(K / \rho \cdot L_{n-p}\right) E_{n-p}$, then

$$
\left[W_{p}(K) / \gamma\right]^{1 /(n-p)} E_{n-p}=\dot{M}_{n-p}^{\rho}(C) .
$$

In turn, by the remark following (4.2)

$$
C(\rho)=\dot{M}_{n-p}^{\rho}\left(\rho^{\prime} \cdot\left[K / \rho \cdot L_{n-p}\right]\right)
$$

where $\rho^{\prime}$ is in that rotation subgroup $\mathscr{R}_{n-p}^{\prime}$ of $\mathscr{R}_{n}$ each element of which transforms $\rho \cdot L_{n-p}$ into itself. For this reason we may write $\rho^{\prime} \cdot K / \rho \cdot L_{n-p}$ for $\rho^{\prime} \cdot\left[K / \rho \cdot L_{n-p}\right]$. Taken together, (4.3) and (4.4) yield

$$
\left[W_{p}(K) / \gamma\right]^{1 /(n-p)} E_{n-p}=\dot{M}_{n-p}^{\rho}\left(\dot{M}_{n-p}^{\rho^{\prime}}\left(\rho^{\prime} \cdot K / \rho \cdot L_{n-p}\right)\right)
$$

as a representation for the mean cross-sectional measures when $p=1,2, \ldots, n-1$. The constant $\gamma$ is easily seen to be $W_{r}(E)=\kappa_{n}$.

5. We begin this section with a list of seven properties of the means $M_{p}$ and $\dot{M}_{p}$ which are analogues of the characterizing properties (1) through (6), listed in $\S 1$, of power means of nonnegative valued functions over $T_{1}$. In each case the analogue is the rather obvious one except that for property (6) there are two equally natural analogues, both of which are listed.

We use $M$ to signify either $M_{p}$ or $\dot{M}_{p}$ for some fixed $p$. The domain of definition $D_{p}$ of $M$ is the set of all bounded, measurable families of convex bodies in $R_{n}$, except in property (II) a somewhat wider class of families is considered. The proof of those properties which are marked with an asterisk will not depend on the 
special structure of $T_{1}$; these properties are valid with $T$ written for $T_{1}$, except (III) must be modified as noted.

(I)* $M$ is continuous; that is if $\left\{\mathbf{K}_{j}\right\}$ is a bounded sequence of families in $D_{n}$ which converges to a family $K$ in the sense that

$$
\lim _{j \rightarrow \infty} \delta\left(K_{j}(t), K(t)\right)=0 \text { for each } t \text { in } T_{1},
$$

then $K$ is in $D_{n}$ and $\lim _{j \rightarrow \infty} M\left(\mathbf{K}_{j}\right)=M(\mathbf{K})$. Here the boundedness of the sequence $\left\{\mathbf{K}_{j}\right\}$ means that there are positive numbers $b_{0}$ and $b_{1}$ such that $b_{0} E \subseteq K_{j}(t) \subseteq b_{1} E$ for all $j$ and all $t$. We revert to the earlier notation of $E$ for $E_{n}$.

(II)* $M$ is monotonic increasing in the sense that $M\left(\mathbf{K}^{\prime}\right) \subseteq M(\mathbf{K})$ whenever $K^{\prime}(t) \subseteq K(t)$ over $T_{1}$. We assume $\mathbf{K}^{\prime}$ is in $D_{m}, \mathbf{K}$ is in $D_{n}$; the possibility that $m<n$ is specifically included.

(III)* $M$ is symmetric in the following sense. Suppose $\mathbf{K}$ is in $D_{n}$. As in (3) set $K^{\prime}(t)=K(t+\tau)$ where' $\tau$ is some fixed element of $T_{1}$, the addition being modulo one. $\mathbf{K}^{\prime}$ is in $D_{n}$ and $M\left(\mathbf{K}^{\prime}\right)=\dot{M(K)}$.

Note. If $T_{1}$ is replaced by a general, compact topological group $T$, then $t+\tau$ is to be understood as the composition of $t$ with $\tau$ in either order. Commutativity is not assumed, of course.

(IV)* If $K(t)=E$ over $T_{1}$, then $M(\mathbf{K})=E$.

(V)* $M$ has the composition property for families $\mathbf{K}$ in $D_{1}$. By this we mean that, in the notation of condition (5), if $C\left(t^{\prime}\right)=K(t), M(\mathbf{C})=C$ and $K^{\prime}(t)=C$ over $T_{1}^{\prime}$ while $K^{\prime}(t)=K(t)$ otherwise, then $M\left(\mathbf{K}^{\prime}\right)=M(\mathbf{K})$. It must be noted that $\mathbf{K}$ is in $D_{1}$; necessarily this is true for $\mathbf{C}$ and $\mathbf{K}^{\prime}$.

(VIa) $M$ is positively homogeneous of degree one; that is, for every constant $\lambda>0$, we have $M(\lambda \mathbf{K})=\lambda M(\mathbf{K})$ for each $\mathbf{K}$ in $D_{n}$.

(VIb)* Suppose $\lambda$ is a real-valued, measurable function over $T_{1}$ satisfying $0<\lambda_{0} \leqq \lambda(t) \leqq \lambda_{1}$. If $K$ is a convex body and $K(t)=\lambda(t) K$, then $\mathbf{K}$ is in $D_{n}$ and $M(\mathbf{K})=\mu K$ for some constant $\mu>0$.

Certain of these properties follow immediately from the representations (3.2) and (3.3) or (3.5) of the means $M_{p}$ and $\dot{M}_{p}$, and the correspondingly numbered properties from the set (1) through (6). This surely applies to (III), (IV), and (VIa). With respect to the more general form of (III), in which $T$ replaces $T_{1}$, we need only remark that the translation-invariant character of the Haar measure insures that (3) is Valid for nonnegative valued functions over $T$.

Property (I) requires somewhat more discussion. Let $H_{j}(x, t)$ and $H(x, t)$ be the support functions of $K_{j}(t)$ and $K(t)$. The convergence of $\left\{\mathbf{K}_{j}\right\}$ to $\mathbf{K}$ means that for each $t$ we have $\lim _{j \rightarrow \infty} H_{j}(x, t)=H(x, t)$ uniformly for $x$ in $\partial E$.

In discussing the deviation $\delta$ in the second section, we noted that this is equivalent to the assertion that, for each $t, \lim _{j \rightarrow \infty} F_{j}(x, t)=F(x, t)$ uniformly for $x$ in $\partial E$, where $F_{j}(x, t)$ and $F(x, t)$ are the distance functions of $K_{j}(t)$ and $K(t)$. For each $x$, $F(x, t)$ is necessarily measurable over $T_{1}$, cf. $\left[8\right.$, p. 84] and $0<1 / b_{1} \leqq F(x, t) \leqq 1 / b_{0}$ 
for $x$ in $\partial E$. This insures the existence of the mean $M_{-p}^{t}(F(x, t))$ and, by Lebesgue's bounded convergence theorem, we have

$$
\lim _{j \rightarrow \infty} M_{-p}^{t}\left(F_{j}(x, t)\right)=M_{-p}^{t}(F(x, t)) .
$$

This convergence is uniform for $x$ in $\partial E$. Hence, by the first part of Theorem 2, the sequence of associated star bodies $\left\{m_{p}\left(\mathbf{K}_{j}\right)\right\}$ converges to $m_{p}(\mathbf{K})$. We now apply the second part of Theorem 2 and conclude that this is true for the convex closures, i.e., $\lim _{j \rightarrow \infty} \dot{M}_{p}\left(\mathbf{K}_{j}\right)=\dot{M}_{p}(\mathbf{K})$.

To complete the proof of property (I), we observe that the preceding argument can be applied again with the functions $H_{j}(x, t), H(x, t)$ viewed as distance functions of the polar reciprocal bodies $\hat{K}_{j}(t), \hat{K}(t)$. For the measurability of the families we appeal to Theorem 3 . Hence $\lim _{j \rightarrow \infty} \dot{M}_{-p}\left(\hat{\mathbf{K}}_{j}\right)=\dot{M}_{-p}(\hat{\mathbf{K}})$. In turn, this implies by polar reciprocation and (3.3) that $\lim _{j \rightarrow \infty} M_{p}\left(\mathbf{K}_{j}\right)=M_{p}(\mathbf{K})$.

Although this settles the continuity property (I), it is convenient at this point to call attention to a more inclusive continuity result. Suppose $\left\{\mathbf{K}_{j}\right\}$, $\mathbf{K}$ have the same meaning as before and that $\left\{p_{j}\right\}$ is a sequence of real numbers which converges to $p$. Then

$$
\lim _{j \rightarrow \infty} \dot{M}_{p_{j}}\left(\mathbf{K}_{j}\right)=\dot{M}_{p}(\mathbf{K}), \quad \lim _{j \rightarrow \infty} M_{p_{j}}\left(\mathbf{K}_{j}\right)=M_{p}(\mathbf{K}) .
$$

The proof is much the same, but with the alteration that we consider the sequence $\left\{M_{-p_{j}}^{t}\left(F_{j}(x, t)\right)\right\}$ in lieu of (5.1). Since the powers and roots of a nonnegative number depend continuously on that number, no new difficulty presents itself.

Two special cases will be of later use. First, by taking all the families $\mathbf{K}_{j}$ to be $\mathbf{K}$, we conclude that $M_{p}(\mathbf{K})$ and $\dot{M}_{p}(\mathbf{K})$ are continuous in $p$. Second, we take two bounded sequences of convex bodies, say $\left\{K_{0}^{(j)}\right\}$ and $\left\{K_{1}^{(j)}\right\}$ which we suppose converge to convex bodies $K_{0}$ and $K_{1}$. Let $\left\{\theta_{j}\right\}$ be a convergent sequence from $T_{1}$. We define $\mathbf{K}_{j}$ by

$$
K_{j}(t)=K_{0}^{(j)} \text { for } 0 \leqq t \leqq \theta_{j}, \quad K_{j}(t)=K_{1}^{(j)} \text { for } \theta_{j}<t \leqq 1
$$

if $\theta_{j}<1$ and $K_{j}(t)=K_{0}^{(j)}$ for $0 \leqq t \leqq 1$ if $\theta_{j}=1$. In this case we conclude that the elementary means $M_{p}\left(K_{0}, K_{1} ; \vartheta\right)$ and $\dot{M}_{p}\left(K_{0}, K_{1} ; \vartheta\right)$ are continuous in

$$
e=\left(p, \vartheta, K_{0}, K_{1}\right)
$$

in the sense of the metric

$$
d\left(e, e^{\prime}\right)=\left|p-p^{\prime}\right|+\left|\vartheta-\vartheta^{\prime}\right|+\delta\left(K_{0}, K_{0}^{\prime}\right)+\delta\left(K_{1}, K_{1}^{\prime}\right) .
$$

The monotonicity property (II) is simply shown when $\mathbf{K}, \mathbf{K}^{\prime}$ are both families of convex bodies in $D_{n}$. Thus $K^{\prime}(t) \subseteq K(t)$ implies $F^{\prime}(x, t) \geqq F(x, t)$ over $R_{n} \times T_{1}$; by (2)

$$
M_{-p}^{t}\left(F^{\prime}(x, t)\right) \geqq M_{-p}^{t}(F(x, t))
$$

and so, by (3.2) $\dot{M}_{p}\left(\mathbf{K}^{\prime}\right) \subseteq \dot{M}_{p}(\mathbf{K})$. The proof for $M_{p}$ is similar. These proofs fail if $\mathbf{K}^{\prime}$ is in $D_{m}$ where $m<n$ and so we must proceed differently. 
Suppose $L$ is the linear, $m$-dimensional space which contains $\mathbf{K}^{\prime}$; we have for all $t$

$$
K^{\prime}(t) \cap L=K^{\prime}(t) \subseteq K^{\star}(t)=K(t) \cap L
$$

By the preceding argument $M\left(\mathbf{K}^{\prime}\right) \subseteq M\left(\mathbf{K}^{\star}\right)$ and so we need show only that $M\left(\mathbf{K}^{\star}\right) \subseteq M(\mathbf{K})$.

For $M=\dot{M}_{p}$ we have: $y$ is a point of $\dot{M}_{p}\left(\mathbf{K}^{\star}\right)$ if $y$ is in $L$ and $y$ is in the convex closure of those $x$ in $L$ for which $M_{-p}^{t}(F(x, t)) \leqq 1$. Thus $y$ is in $\dot{M}_{p}(\mathbf{K})$ as was to be shown.

If $M=M_{p}$ we argue in another fashion. We begin with a preliminary item. Let $K$ be a convex body, $H$ its support function in $L$, i.e., $H$ is defined over $L$. We extend the domain of definition of $H$ to $R_{n}$ as follows. Let $y$ be any point in $R_{n}, y^{*}$ its projection onto $L$. If $y^{*} \neq 0$, set $u=y^{*} /\left\|y^{*}\right\|$; we set

$$
\begin{array}{ll}
H(y)=H(u)(u, y), & \text { when } y^{*} \neq 0, \\
H(y)=0, & \text { when } y^{*}=0 .
\end{array}
$$

This extension yields the support function of $K$ considered as a degenerate convex body in $R_{n}$, that is: a point $x$ of $R_{n}$ is in $K$ if and only if

$$
(x, v) \leqq H(v) \text { for all } v \text { in } \partial E .
$$

First suppose $x$ satisfies these inequalities. Choosing any $v$ orthogonal to $L$ we get $(x, v)=0$ and so $x$ must be in $L$. We see that $x$ must be in $K$ by restricting $v$ to $\partial E \cap L$.

On the other hand, suppose $x$ is in $K$. If $v$ is not orthogonal to $L$ we write $v=w+(u, v) u$ where $w$ is orthogonal to $L$ and $u=v^{*} /\left\|v^{*}\right\|$. Then

$$
(x, v)=(x, w)+(u, v)(x, u) \leqq H(u)(u, v)=H(v),
$$

since $(x, w)=0$. If $v$ is orthogonal to $L,(x, v)=0=H(v)$. Thus in either case (5.2) is satisfied.

Let $H^{\star}$ be the support function of $\mathbf{K}^{\star}$ in $L$. We extend the domain of $H^{\star}$ to $R_{n} \times T_{1}$ according to (5.1). We form $M_{p}^{t}\left(H^{\star}(x, t)\right)=\Phi(x)$ for $x$ in $L$. The intersection over all $u$ in $\partial E \cap L$ of the half-spaces of $L$ described by $(x, u) \leqq \Phi(u)$ is $M_{p}\left(K^{\star}\right)$. We also extend the domain of $\Phi$ to $R_{n}$ by (5.1). When we keep in mind the convention that $M_{p}(f)=0$ if $f$ vanishes over $T_{1}$ even for $p \leqq 0$, we see that

$$
\Phi(y)=\Phi(u)(u, y)=M_{p}^{t}\left(H^{\star}(u, t)(u, y)\right)=M_{p}^{t}\left(H^{\star}(y, t)\right)
$$

for all $y$ in $R_{n}$ since $(u, y) \geqq 0$. Hence the half-spaces

$$
(x, v) \leqq M_{p}^{t}\left(H^{\star}(v, t)\right), \quad v \text { in } \partial E,
$$

intersect to form $M_{p}\left(\mathbf{K}^{\star}\right)$. 
Every supporting half-space of $K(t)$ contains $K^{\star}(t)$. Therefore we have for the extended support function of $K^{\star}(t)$ :

$$
H^{\star}(y, t) \leqq H(y, t) \text { over } R_{n} \times T_{1} .
$$

Hence

$$
M_{p}^{t}\left(H^{\star}(y, t)\right) \leqq M_{p}^{t}(H(y, t)) \text { over } R_{n} .
$$

This shows that the supporting half-spaces of $M_{p}(\mathbf{K})$ contain $M_{p}\left(\mathbf{K}^{\star}\right)$ and so $M_{p}\left(\mathbf{K}^{\star}\right) \subseteq M_{p}(\mathbf{K})$.

The proof of $(\mathrm{V})$ is not difficult, but its restricted character warrants comment. We have observed in $\S 4$ that the convex bodies which make up a family $\mathbf{K}$ in $D_{1}$ are the intervals $-a(t) \leqq x \leqq b(t)$ where $a, b$ are positive, bounded and measurable over $T_{1}$, and further that $M_{p}(\mathbf{K}), \dot{M}_{p}(\mathbf{K})$ are equal to the interval

$$
-M_{p}^{t}(a(t)) \leqq x \leqq M_{p}^{t}(b(t)) .
$$

Since property (5) holds for means of real-valued functions, (V) is established.

Property (V) does not hold generally for families in $D_{n}$ for $n \geqq 2$. More precisely, we shall show the following. Suppose $\mathbf{K}$ is in $D_{n}$; we form $\mathbf{K}^{\prime}$ as described in (V) and (5). Then, for $n \geqq 2$,

$$
\begin{aligned}
& M_{p}\left(\mathbf{K}^{\prime}\right) \subseteq M_{p}(\mathbf{K}), \quad \text { with equality for } p \geqq 1, \\
& \dot{M}_{p}\left(\mathbf{K}^{\prime}\right) \supseteq \dot{M}_{p}(\mathbf{K}), \quad \text { with equality for } p \leqq-1,
\end{aligned}
$$

and there can be inequality for the other cases.

Consider the second inequality. In the notation of (5), let

$$
\Psi_{0}(x)=\left[\int_{r_{1}^{\prime}} F^{-p}\left(x, t^{\prime}\right) d t^{\prime} /\left(\tau^{\prime}-\tau\right)\right]^{-1 / p}
$$

The set $\dot{M}_{p}(\mathrm{C})=C$ is the convex closure of the set $S$ of points $x$ for which $\Psi_{0}(x) \leqq 1$. Let $\Psi_{1}$ be the distance function of $C$; then

$$
\Psi_{1}(x) \leqq \Psi_{0}(x)
$$

since $S \subseteq C$. There can be inequality only if $p>-1$. We now form

$$
\begin{array}{rlrl}
G_{i}(x, t) & =F(x, t), & \text { for } t \text { in } T_{1}-T_{1}^{\prime}, \\
& =\Psi_{i}(x), \quad \text { for } t \text { in } T_{1}^{\prime}, \quad i=0,1 .
\end{array}
$$

We have from property (5): $M_{-p}\left(G_{0}\right)=M_{-p}(F)$, and from (5.5):

$$
M_{-p}\left(G_{0}\right) \geqq M_{-p}\left(G_{1}\right) .
$$

The means $\dot{M}_{p}\left(\mathbf{K}^{\prime}\right)$ and $\dot{M}_{p}(\mathbf{K})$ are respectively the convex closures of the sets points $x$ for which $M_{-p}^{t}\left(G_{1}(x, t)\right) \leqq 1$ and $M_{-p}^{t}\left(G_{0}(x, t)\right) \leqq 1$, from which (5.4) follows with the possibility of inequality only if $p>-1$.

That the latter possibility can be realized is shown by the following example involving elementary means. Let $K$ be the convex closure of the points $( \pm 1,0)$, 
$(0, \pm a)$ of $R_{2}$ where $0<a<1$. Take $\rho$ to be a rotation of the plane about the origin through $\pi / 4$ radians. We form

$$
\begin{aligned}
& K_{0}=\dot{M}_{p}\left(K, \rho^{2} \cdot K ; 1 / 2\right), \quad K_{1}=\dot{M}_{p}\left(K_{0}, \rho \cdot K ; 1 / 3\right), \\
& K_{2}=\dot{M}_{p}\left(K, \rho \cdot K, \rho^{2} \cdot K ; 1 / 3,1 / 3,1 / 3\right) .
\end{aligned}
$$

A direct computation shows that if $x_{i}$ is on $\partial K_{i}, i=1,2$ and also on the ray from $(0,0)$ through $(1,1)$, then

$$
\left\|x_{1}\right\|=\left[\left(1+a_{1}\right) / 3\right]^{1 / p}>\left[\left(1+a_{2}\right) / 3\right]^{1 / p}=\left\|x_{2}\right\|
$$

where

$$
a_{1}=\left(a^{p}+1\right) / 2^{p / 2}>2^{1+p / 2} a^{p} /\left(1+a^{p}\right)=a_{2}
$$

when $p>-1$. Thus $K_{2} \supset K_{1}$.

The inequality (5.3) can be treated by similar arguments.

As for condition (VIb), take $H(x)$ and $F(x)$ to be the support functions of $K$; then those of $K(t)$ are $\lambda(t) H(x)$ and $F(x) / \lambda(t)$ respectively. Therefore

$$
M_{p}^{t}(H(x, t))=H(x) M_{p}^{t}(\lambda(t)), \quad M_{-p}^{t}(F(x, t))=F(x) / M_{p}^{t}(\lambda(t)) .
$$

These two functions satisfy (a), (b), (c') and so, regardless of the value of $p$, they are the support and distance functions of $M_{p}(\mathbf{K})$ and $\dot{M}_{p}(\mathbf{K})$. Consequently we have

$$
M_{p}(\mathbf{K})=\dot{M}_{p}(\mathbf{K})=\mu K, \quad \mu=M_{p}^{t}(\lambda(t)) .
$$

The remainder of this section will be devoted principally to a characterization of the power means $M_{p}$ and $\dot{M}_{p}$. Let us regard these means as functions which associate with each member of $D_{n}$ a convex body in $R_{n}$, for $n=1,2, \ldots$. These functions satisfy conditions (I) through (VIb). The characterization we have in mind is this. Suppose $M$ is any function which associates with each member of $D_{n}$ a convex body in $R_{n}$ for $n=1,2, \ldots$ Further, assume $M$ satisfies conditions (I) through (VIb). Then there is a real number $p$ such that, for each $n$ and each family K in $D_{n}$ we have: $\dot{M}_{p}(\mathbf{K}) \subseteq M(\mathbf{K}) \subseteq M_{p}(\mathbf{K})$. In this light, the means $M_{p}$ and $\dot{M}_{p}$ appear as the extremal functions among those considered. In a subsequent section we shall construct examples of means of an intermediate nature.

As a beginning we give a direct proof that, for a bounded, measurable family $\mathbf{K}$ of convex bodies over $T$, we have

$$
\dot{M}_{p}(\mathbf{K}) \subseteq M_{p}(\mathbf{K}) .
$$

Let $H(x, t), F(x, t)$ be the support and distance functions of $K(t)$. Choose $v$ in $\partial E$ and let $x(t)$ be the intersection of the ray $R_{v}$ with $\partial K(t)$. If $y \neq 0$ is on $R_{v}$, we have $F(y, t)=\|y\| /\|x(t)\|$ and so

$$
M_{-p}^{t}(F(x, t))=\|y\| / M_{p}^{t}(\|x(t)\|) .
$$

$m_{p}(\mathbf{K})$ is the star body whose distance function is $M_{-p}(F)$; we choose $y$ to be the 
intersection of $R_{v}$ with $\partial m_{p}(\mathbf{K})$. Consequently $M_{-p}^{t}(F(y, t))=1$ and so

$$
\|y\|=M_{p}^{t}(\|x(t)\|) \text {. }
$$

It follows that, for any vector $u$,

$$
(y, u)=\|y\|(u, v)=\operatorname{sgn}(u, v) M_{p}^{t}(|(x(t), u)|) .
$$

By our choice of $x$ we have

$$
(x(t), u)=\operatorname{sgn}(u, v)|(x(t), u)| \leqq H(u, t),
$$

therefore $(y, u) \leqq M_{p}^{t}(H(u, t))$ for all $u$ in $\partial E$.

Thus $y$ is a point of $M_{p}(\mathbf{K})$ and so we have $m_{p}(\mathbf{K}) \subseteq M_{p}(\mathbf{K})$. We take convex closures on both sides; since $\bar{m}_{p}(\mathbf{K})=\dot{M}_{p}(\mathbf{K})$ this gives (5.7).

Inequality (5.7) will not be used in the proof of the characterization theorem; that theorem gives a second proof for the special case of families in $D_{n}$.

Some cases of equality in (5.7) can be described easily: there is equality if, for some convex body $K, K(t)=\lambda(t) K$ almost everywhere in $T$.

We shall call $M$ a power mean if it associates with each $n$ and each $\mathbf{K}$ in $D_{n}$ a convex body $M(\mathrm{~K})$ in $R_{n}$ in such a way that conditions (I) through (VIb) are fulfilled.

Let us first consider means of families $\mathbf{K}$ in $D_{1}$; as we have noted earlier $K(t)$ is the interval

$$
-a(t) \leqq x \leqq b(t)
$$

where $a, b$ are bounded, measurable, positive functions which are bounded away from zero. We now prove:

THEOREM 6. If $\mathbf{K}$ is the bounded, measurable family of convex bodies in $R_{1}$ described by (5.8) and $M$ is a power mean, then $M(\mathbf{K})$ is the interval

$$
-M_{p}^{t}(a(t)) \leqq x \leqq M_{p}^{t}(b(t))
$$

for some index $p$ which is independent of $\mathbf{K}$.

First suppose $\mathbf{K}$ has the special form

$$
K(t)=a(t) K
$$

where $K$ is the closed interval $-1 \leqq x \leqq c$, for some $c>0$. By property (VIb), $M(\mathbf{K})$ is the interval $-\mu \leqq x \leqq \mu c$, where $\mu=\mu(a(t))$ is a functional depending only on $a(t)$. We claim that $\mu$ satisfies conditions (1) through (6). When the families $\mathbf{K}, \mathbf{K}_{j}, \mathbf{K}^{\prime}$ in $D_{1}$ are of the restricted type (5.9), we see that the following assertions are true over $T_{1}:\left\{\mathbf{K}_{j}\right\}$ converges to $\mathbf{K}$ if and only if $\left\{a_{j}(t)\right\}$ converges to $a(t) ; K^{\prime}(t) \subseteq K(t)$ if and only if $a^{\prime}(t) \leqq a(t) ; \lambda \mathbf{K}$ is the family determined by $\lambda a(t)$ when $\mathbf{K}$ is determined by $a(t) ; K(t)=K$ for some fixed convex body $K$ if and only if $a(t)$ is constant. With the aid of these observations and the assumption that $M(\mathbf{K})$ satisfies (I) through (VIb), it is routine to verify that $\mu$ satisfies (1) through (6). 
Therefore, by the characterization theorem for power means of functions in $D$, we conclude that $\mu(a(t))=M_{p}^{t}(a(t))$ for some real index $p$ which does not depend on $a(t)$. Consequently, if $\mathbf{K}$ is of the special form (5.9), then $M(\mathbf{K})$ is the interval

$$
-M_{p}^{t}(a(t)) \leqq x \leqq c M_{p}^{t}(a(t)) .
$$

In view of property (6) applied to the right-hand side of the preceding inequality, we see that the theorem is true for families of the special form (5.9).

Now consider a family in $D_{1}$ of the general form (5.8). Let $c_{i}, i=0,1$, be positive constants such that

$$
c_{0} a(t) \leqq b(t) \leqq c_{1} a(t)
$$

over $T_{1}$. We define the families $\mathbf{K}_{i}$ to be the intervals

$$
K_{i}(t):-a(t) \leqq x \leqq c_{i} a(t) .
$$

From (5.10) it follows that $K_{0}(t) \subseteq K(t) \subseteq K_{1}(t)$ over $T_{1}$ and so, by property (II), $M\left(\mathbf{K}_{0}\right) \subseteq M(\mathbf{K}) \subseteq M\left(\mathbf{K}_{1}\right)$.

Since $\mathbf{K}_{i}$ are of the special form (5.9), the sets $M\left(\mathbf{K}_{i}\right)$ are the intervals

$$
-M_{p}^{t}(a(t)) \leqq x \leqq c_{i} M_{p}^{t}(a(t)) .
$$

Therefore, if $M(\mathbf{K})$ is the interval: $-\alpha \leqq x \leqq \beta$, then, by using (II), we conclude that $\alpha=M_{p}^{t}(a(t))$. Hence the left end point of $M(\mathbf{K})$ has the form asserted in the theorem. We interchange the roles of left and right boundaries, repeat the argument and thereby complete the proof of the theorem.

Let $M$ be a power mean; we define the conjugate of $M$ to be $M^{\prime}$ described by $M^{\prime}(\mathbf{K})=\hat{M}(\hat{\mathbf{K}})$ where $\mathbf{K}$ is in $D_{n}$. It is clear from Theorem 3 that $M^{\prime}$ is defined for all $\mathbf{K}$ in $D_{n}$.

THEOREM 7. If $M$ is a power mean, so is its conjugate $M^{\prime}$. The conjugate of $M^{\prime}$ is $M$.

The last assertion is a direct consequence of the involutory nature of polar reciprocation, viz.

$$
M^{\prime \prime}(\mathbf{K})=\left[M^{\prime}(\hat{\mathbf{K}})\right]^{\wedge}=\hat{M}\left([\hat{\mathbf{R}}]^{\wedge}\right)^{\wedge}=M(\mathbf{K}) .
$$

We must show that $M^{\prime}$ satisfies conditions (I) through (VIb).

I. If $\left\{\mathbf{K}_{j}\right\}$ has limit $\mathbf{K}$, then $\left\{\hat{\mathbf{K}}_{j}\right\}$ has limit $\hat{\mathbf{K}}$ because the hypothesis implies the convergence of the support functions $H_{j}(x, t)$ to $H(x, t)$, uniformly over $x$ in $\partial E$ as $j$ tends to infinity for each $t$ in $T_{1}$. In turn, this implies the same sort of convergence of the distance functions $F_{j}(x, t)$ to $F(x, t)$ by the remarks at the end of \$2. Therefore, since $M$ satisfies (I), $\lim _{j \rightarrow \infty} M\left(\hat{\mathbf{K}}_{j}\right)=M(\hat{\mathbf{K}})$. By the same type of convergence argument applied to the sequence $\left\{M\left(\mathbf{K}_{j}\right)\right\}$, we conclude

$$
\lim _{j \rightarrow \infty} M^{\prime}\left(\mathbf{K}_{j}\right)=\lim _{j \rightarrow \infty} \hat{M}\left(\hat{\mathbf{K}}_{j}\right)=\hat{M}(\hat{\mathbf{K}})=M^{\prime}(\mathbf{K}) .
$$


II. If $K^{\prime}(t) \subseteq K(t)$ over $T_{1}$, then $\hat{K}^{\prime}(t) \supseteq \hat{K}(t)$, and so $M\left(\hat{\mathbf{K}}^{\prime}\right) \supseteq M(\hat{\mathbf{K}})$ by property (II). Take polar reciprocals to finish the proof.

Conditions (III) and (IV) are immediate.

V. We begin with a family $\mathbf{K}$ in $D_{1}$ of the form (5.8). Then $\hat{K}(t)$ is the interval $-1 / a(t) \leqq x \leqq 1 / b(t)$, and so, by Theorem $6, M(\hat{\mathbf{K}})$ is the interval

$$
-M_{p}^{t}(1 / a(t)) \leqq x \leqq M_{p}^{t}(1 / b(t)) \text {. }
$$

Therefore, by taking polar reciprocals, we find that $M^{\prime}(\mathbf{K})$ is the interval

$$
-M_{-p}^{t}(a(t)) \leqq x \leqq M_{-p}^{t}(b(t))
$$

Since $M_{-p}$ as a mean of functions over $D$ enjoys the composition property (5), $M^{\prime}(\mathbf{K})$ has the composition property (V).

VIa. If $K^{\prime}(t)=\lambda K(t)$ for a constant $\lambda>0$, then $\hat{K}^{\prime}(t)=(1 / \lambda) \hat{K}(t)$. Consequently

$$
M^{\prime}\left(\mathbf{K}^{\prime}\right)=[(1 / \lambda) M(\hat{\mathbf{K}})]^{\wedge}=\lambda \hat{M}(\hat{\mathbf{K}})=\lambda M^{\prime}(\mathbf{K}) .
$$

VIb. The proof is similar to that of (VIa); we begin with $\hat{K}(t)=(1 / \lambda(t)) \hat{K}$ and so $M(\hat{\mathbf{K}})=\left(1 / \mu^{\prime}\right) \hat{K}$ for some constant $\mu^{\prime}>0$ by property (VIb). Thus

$$
\hat{M}(\hat{\mathbf{K}})=M^{\prime}(\mathbf{K})=\mu^{\prime} K
$$

This completes the proof of Theorem 7. By way of example, (3.3) asserts that the conjugate of the power mean $\dot{M}_{-p}(\mathbf{K})$ is $M_{p}(\mathbf{K})$.

As further preparation for the characterization theorem, we shall establish the following inequalities, valid for any power mean $M . \mathbf{K}$ is in $D_{n}$ and $L$ is a linear subspace of $R_{n}$. In the notation of (4.1):

$$
\begin{aligned}
& M^{\star}(\mathbf{K}) \supseteq M\left(\mathbf{K}^{\star}\right), \\
& M^{*}(\mathbf{K}) \subseteq M\left(\mathbf{K}^{*}\right) .
\end{aligned}
$$

From the monotonicity property (II) of $M$ we have $M(\mathbf{K}) \supseteq M\left(\mathbf{K}^{\star}\right)$ and $M\left(\mathbf{K}^{\star}\right)$ is in $L$. Hence $M^{\star}(\mathbf{K}) \supseteq\left[M\left(\mathrm{~K}^{\star}\right)\right]^{\star}=M\left(\mathrm{~K}^{\star}\right)$, which proves (5.11).

Suppose $K$ is a convex body in $R_{n}$ and $H$ is its support function; then $(\hat{K})^{\star}$ has a distance function which is the restriction of $H$ to points in $L$. Consequently

$$
\left(K^{*}\right)^{\wedge}=(\hat{K})^{\star} \text {. }
$$

A similar argument applied to the distance function of $K$ shows that

$$
\left(K^{\star}\right)^{\wedge}=(\hat{K})^{*} \text {. }
$$

The polar reciprocations on the left are with respect to the unit sphere in $L$, viz. $E^{*}=E^{\star}$

We use these relations to deduce (5.12) from (5.11) by a polar reciprocation argument. By Theorem 7 we may introduce the conjugate mean $M^{\prime}$ and, from (5.11) applied to the family $\hat{\mathbf{K}}$, we have $\left[M^{\prime}(\hat{\mathbf{K}})\right]^{\star} \supseteq M^{\prime}\left([\hat{\mathbf{K}}]^{\star}\right)$. Taking polar reciprocals, we reverse this inequality and obtain $\left(\left[M^{\prime}(\hat{\mathbf{R}})\right]^{\star}\right)^{\wedge} \subseteq \hat{M}^{\prime}\left([\mathbf{R}]^{\star}\right)$. Apply 
(5.13) and (5.14); this gives $\left[\hat{M}^{\prime}(\hat{\mathbf{K}})\right]^{*} \subseteq \hat{M}^{\prime}\left(\left[\mathbf{K}^{*}\right]^{\wedge}\right)$ which is, according to the latter part of Theorem 7 , just (5.12).

Consider the family $\mathbf{K}$ where $K(t)=\lambda(t) E$. By (5.11) and (5.12) we have

$$
M^{\star}(\lambda(t) E) \supseteq M\left(\lambda(t) E^{\star}\right)=M\left(\lambda(t) E^{*}\right) \supseteq M^{*}(\lambda(t) E) .
$$

On the other hand, from property (VIb),

$$
M^{\star}(\lambda(t) E)=\mu E^{\star}=\mu E^{*}=M^{*}(\lambda(t) E)
$$

for some constant $\mu>0$. Hence

$$
M^{\star}(\lambda(t) E)=M\left(\lambda(t) E^{\star}\right)=\mu E^{\star}
$$

for any linear subspace $L$.

One important consequence of (5.15) is this: if $L$ is a one-dimensional subspace, then $\mu$ has the form $\mu=M_{p}^{t}(\lambda(t))$ by Theorem 6 . The index $p$ is independent of the choice of $L$ since this is true of $\mu$. This permits us to define an index of any power mean $M$; we take it to be $p$ when this is the index of $M$ for families in $D_{1}$ and for the special families $\lambda(t) E$ in $D_{n}$. Of course, this agrees with the indexing of $M_{p}$ and $\dot{M}_{p}$ in view of (5.6). When we have finished the characterization theorem, it will follow that $\mu$ will have the form indicated for all the families considered in (VIb).

THEOREM 8. If $M$ is a power mean and its index is $p$, then $\dot{M}_{p}(\mathbf{K}) \subseteq M(\mathbf{K}) \subseteq M_{p}(\mathbf{K})$ for each bounded, measurable family $K$ of convex bodies. There is equality on both sides if $K(t)=\lambda(t) K$ almost everywhere in $T_{1}$ for some convex body $K$.

Let $L$ be an arbitrary, one-dimensional, linear subspace. By Theorem 6

$$
M\left(\mathbf{K}^{\star}\right)=\dot{M}_{p}\left(\mathbf{K}^{\star}\right)
$$

since $M$ has index $p$.

We next show that

$$
\dot{M}_{p}(\mathbf{K})=\varpi \dot{M}_{p}\left(\mathbf{K}^{\star}\right)
$$

where, throughout this proof, unions are to be taken over all choices of $L$. Indeed, $\dot{M}_{p}\left(\mathbf{K}^{\star}\right)$ is the interval of points $x$ in $L$ for which $M_{-p}^{t}(F(x, t)) \leqq 1$ where $F(x, t)$ is the distance function of $K(t)$ and, consequently, that of $K^{\star}(t)$ when $x$ is restricted to $L$. By definition $\dot{M}_{p}(\mathbf{K})$ is the convex closure of that star body which is the union of such intervals. Thus (5.17) is established.

From inequality (5.11) we have

$$
M(\mathbf{K})=\bar{\bigcup} M^{\star}(\mathbf{K}) \supseteq \bigcup M\left(\mathbf{K}^{\star}\right)=\bar{\bigcup} \dot{M}_{p}\left(\mathbf{K}^{\star}\right) ;
$$

the final equality comes from (5.16). We couple this with (5.17) and obtain, for any $\mathbf{K}$ in $D_{n}$,

$$
M(\mathbf{K}) \supseteq \dot{M}_{p}(\mathbf{K})
$$


To obtain the remaining inequality, let $M^{\prime}$ be the conjugate mean to $M ; M^{\prime}$ has index $-p$ and so, by $(5.18), M^{\prime}(\hat{\mathbf{K}}) \supseteq \dot{M}_{-p}(\hat{\mathbf{K}})$ for any $\hat{\mathbf{K}}$ in $D_{n}$. Taking polar reciprocals reverses this inequality; we then use the latter part of Theorem 7 and (3.14) to get $M(\mathbf{K}) \subseteq M_{p}(\mathbf{K})$ for the family $\mathbf{K}$ in $D_{n}$.

It has been noted already in (5.6) that, when $\mathbf{K}$ has the special form $K(t)=\lambda(t) K$ for almost all $t$, then $M_{p}(\mathbf{K})=\dot{M}_{p}(\mathbf{K})=M_{p}^{t}(\lambda(t)) K$. The inequalities just established show that for such families we have $M_{p}(\mathbf{K})=M(\mathbf{K})=\dot{M}_{p}(\mathbf{K})=M_{p}^{t}(\lambda(t)) K$ for any power mean $M$. In particular, as a generalization of property (VI) we have: if $K(t)=K$ over $T_{1}$ for some fixed convex body $K$, then $M(\mathbf{K})=K$.

We close this section with a comment on condition (VIb). The condition was used twice: first to prove Theorem 6 and second to prove (5.15). In the first case, (VIb) is only required to hold for all families $\mathbf{K}$ of the special form in $D_{1}$; in the second case, for all families of concentric spheres in $D_{n}, n=1,2, \ldots$ Thus we need only assume: (VIb'). Suppose $\mathbf{K}$ is a family in $D_{n}$ of the special form $K(t)=\lambda(t) K$ where $K$ is a fixed convex body. Then $M(\mathbf{K})=\mu K$ for some $\mu>0$ if (a) $n=1$, or (b) $K=E, n=1,2, \ldots$

On the basis of those cases of equality mentioned in Theorem 8 , we see that necessarily $M$ satisfies (VIb) whenever it satisfies (I) through (VIb').

6. This section is devoted to analogues of the inequalities of Jensen and Jessen.

For Jensen's inequality we have:

$$
M_{p}(\mathbf{K}) \subseteq M_{q}(\mathbf{K}), \quad \dot{M}_{p}(\mathbf{K}) \subseteq \dot{M}_{q}(\mathbf{K}) \quad \text { whenever } p \leqq q
$$

for any family in $D_{n}, n=1,2, \ldots$ The proof is immediate. Let $H(x, t)$ be the support function of $K(t)$, then $M_{p}^{t}(H(x, t)) \leqq M_{q}^{t}(H(x, t))$ over $R_{n}$ by Jensen's inequality (1.2). In view of the partial ordering of convex bodies induced by that of their support functions, mentioned in $\$ 1$, this last inequality is equivalent to the first half of (6.1). If $F(x, t)$ is the distance function $K(t)$, then, since $-p \geqq-q$, $M_{-p}^{t}(F(x, t)) \geqq M_{-q}^{t}(F(x, t))$. By referring to the partial ordering of convex bodies induced by that of their distance functions, we establish the latter part of (6.1).

Because bounded, monotonic sequences of convex bodies have unique limits in the sense of the deviation $\delta$, cf. [10, p. 152], and because from $b_{0} E \subseteq K(t) \subseteq b_{1} E$ we deduce

$$
b_{0} E \subseteq \dot{M}_{p}(\mathbf{K}) \subseteq M_{p}(\mathbf{K}) \subseteq b_{1} E,
$$

the inequalities (6.1) suggest the examination of the limits of the means $M_{p}(\mathbf{K})$, $\dot{M}_{p}(\mathbf{K})$ as $p$ tends to $\pm \infty$.

To this end we define two convex bodies $\gamma(\mathbf{K})$ and $\Gamma(\mathbf{K})$ associated with a bounded, measurable family $\mathbf{K}$ over $T$ in the following way. Let $C$ be a convex body such that

$$
K(t) \subseteq C
$$


for almost all $t$. The intersection of all such sets is $\Gamma(\mathbf{K})$. In a similar fashion, let $c$ be a convex body such that $c \subseteq K(t)$ for almost all $t$. The convex closure of the union of all such sets $c$ is $\gamma(\mathbf{K})$. From the order-reversing character of polar reciprocation and from $\bar{U} \hat{C}=(\cap C)^{\wedge}$ it is seen that

$$
\gamma(\hat{\mathbf{K}})=\hat{\Gamma}(\mathbf{K}) .
$$

We claim that

$$
\lim _{p \rightarrow \infty} M_{p}(\mathbf{K})=\lim _{p \rightarrow \infty} \dot{M}_{p}(\mathbf{K})=\Gamma(\mathbf{K}), \lim _{p \rightarrow-\infty} M_{p}(\mathbf{K})=\lim _{p \rightarrow-\infty} \dot{M}_{p}(\mathbf{K})=\gamma(\mathbf{K}) .
$$

In virtue of inequality (5.7) and the monotonic character of $M_{p}$ and $\dot{M}_{p}$ in $p$, only the two right-hand equalities must be shown; further, equations (6.3) and (3.3) show that the second of these follows from the first.

If $\Phi$ is the distance function of $C$, by (6.2) we have $F(x, t) \geqq \Phi(x)$ for almost all $t$ and for each $x$ in $R_{n}$. The value of the distance function of the intersection of such sets $C$ at $x$ is $\sup \{\Phi(x)\}$; this is to say, the distance function of $\Gamma(\mathbf{K})$ has eff $\sup F(x, t)$ as its value at $x$. By the convention mentioned in $\S 1$, we conclude that the distance function of $\Gamma(\mathbf{K})$ is $M_{-\infty}^{t}(F(x, t))=\lim _{p \rightarrow \infty} M_{-p}^{t}(F(x, t))$ which gives the desired equality.

In case $\mathbf{K}$ is piecewise constant and if $\left\{K_{j}\right\}, j=0,1, \ldots, m$, is its range, then

$$
\Gamma(\mathbf{K})=\bigcup_{j} K_{j}, \quad \gamma(\mathbf{K})=\bigcap_{j} K_{j} .
$$

For Jessen's inequality we are concerned with a family $K$ of convex bodies in $R_{n}$ defined over $T \times T^{\prime}$ where $T, T^{\prime}$ denote, as usual, compact topological groups with Haar measures so normalized that $T$ and $T^{\prime}$ each have total measure one. The Haar measure for $T \times T^{\prime}$ is the product measure. We assume $K$ is a bounded family in the same sense as before and that it is measurable over $T \times T^{\prime}$. By definition the distance function $F\left(x, t, t^{\prime}\right)$ of $\mathbf{K}$ is measurable over $T \times T^{\prime}$, and, by Fubini's theorem, the means $M_{-p}^{t}\left(F\left(x, t, t^{\prime}\right)\right)$ exist for almost all values of $t^{\prime}$ in $T^{\prime}$. For such $t^{\prime}$ we form

$$
C\left(t^{\prime}\right)=\dot{M}_{p}^{t}\left(K\left(t, t^{\prime}\right)\right),
$$

that is the convex closure of the set of points $x$ in $R_{n}$ for which $M_{-p}^{t}\left(F\left(x, t, t^{\prime}\right)\right) \leqq 1$. Over the remaining set of $t^{\prime}$-values, which is of zero measure, we may set $C\left(t^{\prime}\right)=K_{0}$ where $K_{0}$ is some convex body lying between the bounds for $\mathbf{K}$. In this way we obtain a family of convex bodies $\mathbf{C}$ over $T^{\prime}$ which is bounded and measurable according to Theorem 4 taken together with Fubini's theorem. Thus we may form $\dot{M}_{q}^{t^{\prime}}(\mathbf{C})=\dot{M}_{q}^{t^{\prime}} \dot{M}_{p}^{t}(\mathbf{K})$; clearly the choice of $K_{0}$ does not affect the determination of this mean.

In like fashion we may form $\dot{M}_{p}^{t} \dot{M}_{q}^{t^{\prime}}(\mathbf{K})$.

Let us consider the family $\hat{\mathbf{K}}$ and take $\mathbf{C}$ to be defined as before except that in (6.5) we write $\hat{\mathbf{R}}$ for $\mathbf{K}$. By the corollary to Theorem $3, \hat{\mathbf{R}}$ and $\mathbf{C}$ are bounded, 
measurable families; according to (3.3), the family $\widehat{\mathbf{C}}$ is described by

$$
\hat{C}\left(t^{\prime}\right)=M_{-p}^{t}\left(K\left(t, t^{\prime}\right)\right) \text {. }
$$

We form $\dot{M}_{q}^{t^{\prime}}(\hat{\mathbf{C}})$ and take the polar reciprocal which gives, again by (3.3), $M_{-q}^{t^{\prime}} M_{-p}^{t}(\mathbf{K})$. In the same way we may form $M_{-p}^{t} M_{-q}^{t^{\prime}}(\mathbf{K})$. It is convenient to write $p$ and $q$ for $-p$ and $-q$.

Our analogues of Jessen's inequality will be:

$$
\begin{array}{ll}
M_{q}^{t^{\prime}} M_{p}^{t}(\mathbf{K}) \subseteq M_{p}^{t} M_{q}^{t^{\prime}}(\mathbf{K}) \text { if } p \leqq q, \quad q \geqq 1 ; \\
\dot{M}_{q}^{t^{\prime}} \dot{M}_{p}^{t}(\mathbf{K}) \subseteq \dot{M}_{p}^{t} \dot{M}_{q}^{t^{\prime}}(\mathbf{K}) \text { if } p \leqq q, \quad p \leqq-1 .
\end{array}
$$

It should be noted that the analogy is incomplete: in the first case due to the final restriction on $q$, in the second case due to that on $p$. In fact, we shall show that these inequalities fail to be true generally without some such limitations. We assume the indices to be finite.

The validity of either (6.6) or (6.7) entails the validity of the other. This is shown by a polar reciprocation argument; we shall show that (6.6) implies (6.7) and it will be clear that the same reasoning gives the reversed implication.

Assume (6.6) in the form

$$
M_{-q}^{t^{\prime}} M_{-p}^{t}(\hat{\mathbf{K}}) \supseteq M_{-p}^{t} M_{-q}^{t^{\prime}}(\hat{\mathbf{K}}) \text { for } p \leqq q, \quad p \leqq-1 .
$$

Take polar reciprocals; this reverses the inequality and a double application of (3.4) yields (6.7). It follows also that whenever one of these inequalities fails to be true without the final index restriction, so does the other.

We now prove (6.6). Since $q \geqq 1$, the support function of $M_{q}^{t^{\prime}}\left(K\left(t, t^{\prime}\right)\right)$ is $M_{q}^{t^{\prime}}\left(H\left(x, t, t^{\prime}\right)\right)$ for almost all $t$. We form the half-spaces $h(u)$ described by

$$
(x, u) \leqq M_{p}^{t} M_{q}^{t^{\prime}}\left(H\left(u, t, t^{\prime}\right)\right)
$$

their intersection, over all $u$ in $\partial E$, is $M_{p}^{t} M_{q}^{t^{\prime}}(\mathbf{K})$. We next form, for almost all $t^{\prime}$, the half-spaces $(x, u) \leqq M_{p}^{t}\left(H\left(u, t, t^{\prime}\right)\right)$. Let $H^{\prime}\left(x, t^{\prime}\right)$ be the support function of their intersection over all $u$ in $\partial E$. Then $M_{q}^{t^{\prime}} M_{p}^{t}(\mathbf{K})$ is the intersection, over all $u$ in $\partial E$, of the half-spaces $h^{\prime}(u)$ described by $(x, u) \leqq M_{q}^{t^{\prime}}\left(H^{\prime}\left(u, t^{\prime}\right)\right)$. Because $H^{\prime}\left(u, t^{\prime}\right) \leqq M_{p}^{t}\left(H\left(u, t, t^{\prime}\right)\right)$, for almost all $t^{\prime}$, we have

$$
M_{q}^{t^{\prime}}\left(H^{\prime}\left(u, t^{\prime}\right)\right) \leqq M_{q}^{t^{\prime}} M_{p}^{t}\left(H\left(u, t, t^{\prime}\right)\right) .
$$

By Jessen's inequality, since $p \leqq q$,

$$
M_{q}^{t^{\prime}} M_{p}^{t}\left(H\left(u, t, t^{\prime}\right)\right) \leqq M_{p}^{t} M_{q}^{t^{\prime}}\left(H\left(u, t, t^{\prime}\right)\right)
$$

These last two inequalities imply that $h^{\prime}(u) \subseteq h(u)$ for each $u$ in $\partial E$. From this (6.6) follows by taking intersections over $\partial E$.

The following example is intended to show that (6.7) is not always true if $-1<p$. More precisely, we shall construct a family $\mathbf{K}$ such that if $p, q$ are any two positive numbers satisfying $p<q$, then for this family (6.7) is reversed. Further, there are nonpositive $p>-1$ and choices of $q>p$ for which (6.7) is reversed. 
Let $K_{0}$ be the convex closure of the points $( \pm 1,0),\left(0, \pm \operatorname{ctn} \theta_{0}\right), 0<\theta_{0}<\pi / 2$, in $R_{2}$. Direct computation shows that the distance function $F_{0}$ of $K_{0}$ is defined by

$$
F_{0}(x)=\|x\|\left|\cos \left(\theta-\theta_{0}\right)\right| / \cos \theta_{0}
$$

where $\theta$ is such that $(\cos \theta, \sin \theta)$ is the vector $x /\|x\|$ if $\|x\| \neq 0$. Let $\rho\left(t^{\prime}\right)$ signify a rotation of $R_{2}$ about $(0,0)$ through $2 \pi t^{\prime}$ radians. We define the family $\mathbf{K}$ over the square:

by

$$
0 \leqq t \leqq 1, \quad 0 \leqq t^{\prime} \leqq 1
$$

$$
\begin{aligned}
K\left(t, t^{\prime}\right) & =\rho\left(t^{\prime}\right) \cdot K_{0} \quad \text { for } 0 \leqq t \leqq 1 / 2, \quad 0 \leqq t^{\prime} \leqq 1 ; \\
& =\rho\left(t^{\prime}-1 / 4\right) \cdot K_{0}, \quad \text { for } 1 / 2<t \leqq 1, \quad 0 \leqq t^{\prime} \leqq 1 .
\end{aligned}
$$

The distance function of $K\left(t, t^{\prime}\right)$ is, correspondingly,

$$
\begin{aligned}
F\left(x, t, t^{\prime}\right) & =\|x\|\left|\cos \left(\theta-\theta_{0}-2 \pi t^{\prime}\right)\right| / \cos \theta_{0}, \\
& =\|x\|\left|\cos \left(\theta-\theta_{0}-2 \pi t^{\prime}+\pi / 2\right)\right| / \cos \theta_{0} .
\end{aligned}
$$

The mean $\dot{M}_{q}^{t^{\prime}}\left(K\left(t, t^{\prime}\right)\right)$ is a circle whose radius $r\left(\theta_{0}\right)$ is independent of $t$. Let $e$ be the point $(1,0)$; then the radius is given by

$$
r\left(\theta_{0}\right)=\left(\int_{0}^{1} F^{-q}\left(e, t, t^{\prime}\right) d t^{\prime}\right)^{1 / q}=\cos \theta_{0}\left(\frac{2}{\pi} \int_{-\theta_{0}}^{\pi / 2-\theta_{0}} \cos ^{-q} \psi d \psi\right)^{1 / q}
$$

when $q \neq 0$. Clearly $\dot{M}_{p}^{t} \dot{M}_{q}^{t^{\prime}}(\mathbf{K})$ is this same circle.

We now form $\dot{M}_{p}^{t}\left(K\left(t, t^{\prime}\right)\right)$. From the supposition that $p>-1$, it can be shown with the aid of Minkowski's inequality that this set must be a square with vertices $( \pm c, 0),(0, \pm c)$ where

or

$$
1 / c=\left(\left[F^{-p}\left(e, 0, t^{\prime}\right)+F^{-p}\left(e, 1, t^{\prime}\right)\right] / 2\right)^{-1 / p},
$$

$$
c=\left(\left[1+\operatorname{ctn}^{p} \theta_{0}\right] / 2\right)^{1 / p}=\cos \theta_{0} /\left(\left[\sin ^{-p} \theta_{0}+\cos ^{-p} \theta_{0}\right] / 2\right)^{-1 / p} .
$$

In turn $\dot{M}_{q}^{t^{\prime}} \dot{M}_{p}^{t}(\mathbf{K})$ is a circle of radius $r^{\prime}\left(\theta_{0}\right)$. To find $r^{\prime}\left(\theta_{0}\right)$ we note that in the description of $K_{0}$ we set $\theta_{0}=\pi / 4$, we have a figure similar to $\dot{M}_{p}^{t}\left(K\left(t, t^{\prime}\right)\right)$. From this it follows that $r^{\prime}\left(\theta_{0}\right)=\operatorname{cr}(\pi / 4)$.

Consider $\left[r\left(\theta_{0}\right)\right]^{q}$ in the case $q>0$. By L'Hospital's rule

$$
\begin{aligned}
\lim _{\theta_{0} \rightarrow \pi / 2} r^{q}\left(\theta_{0}\right) & =\lim _{\theta_{0} \rightarrow \pi / 2} \frac{2}{\pi} \int_{-\theta_{0}}^{\pi / 2-\theta_{0}} \cos ^{-q} \psi d \psi / \sec ^{q} \theta_{0} \\
& =\lim _{\theta_{0} \rightarrow \pi / 2} 2\left(\cos ^{-q} \theta_{0}-\sin ^{-q} \theta_{0}\right) / q \sec ^{q} \theta_{0} \tan \theta_{0} \\
& =\lim _{\theta_{0} \rightarrow \pi / 2} 2 \operatorname{ctn} \theta_{0}\left(1-\operatorname{ctn}^{q} \theta_{0}\right) / \pi q=0
\end{aligned}
$$

Hence, for $q>0, \lim _{\theta_{0} \rightarrow \pi / 2} r\left(\theta_{0}\right)=0$. On the other hand $r(\pi / 4)>0$ and, for $p>0$, the first representation of $c$ shows that $\lim _{\theta_{0} \rightarrow \pi / 2} c=2^{-1 / p}$. Thus, by taking $\theta_{0}$ sufficiently near $\pi / 2$, we have $r^{\prime}\left(\theta_{0}\right)>r\left(\theta_{0}\right)$. In short, given $p, q$ such that $0<p<q$, we can choose $\theta_{0}$ so that $\dot{M}_{q}^{t^{\prime}} \dot{M}_{p}^{t}(\mathbf{K}) \supseteq \dot{M}_{p}^{t} \dot{M}_{q}^{t^{\prime}}(\mathbf{K})$. 
The function $g\left(\theta_{0}\right)=\ln \left[r^{\prime}\left(\theta_{0}\right) / r\left(\theta_{0}\right)\right]$ is analytic near $\theta_{0}=\pi / 4$ and, because $c=1$ when $\theta_{0}=\pi / 4, g(\pi / 4)=0$. Direct calculation, with the aid of the second representation of $c$, gives

$$
g\left(\theta_{0}\right)=\left[(p+2)-2^{q / 2} / \int_{0}^{\pi / 4} \cos ^{-q} \psi d \psi\right]\left(\theta_{0}-\pi / 4\right)^{2} / 2+\cdots .
$$

The index $q$ is restricted by $q>-1$; therefore by Jensen's inequality $\pi(2 / 4)^{1 / 2}=\left(4 \int_{0}^{\pi / 4} \cos \psi d \psi / \pi\right)^{-1}<\left(4 \int_{0}^{\pi / 4} \sec ^{q} \psi d \psi / \pi\right)^{1 / q} \leqq \max _{0 \leqq \psi \leqq \pi / 4} \sec \psi=\sqrt{ } 2$.

Consequently

$$
(4 / \pi)^{q+1} \gtreqless 2^{q / 2} / \int_{0}^{\pi / 4} \cos ^{-q} \psi d \psi \gtreqless 4 / \pi, \text { for } q \gtreqless 0 .
$$

We apply this inequality to the leading coefficient in the expansion of $g\left(\theta_{0}\right)$. This shows that if $(4 / \pi)-2<p<q<0$, then, for $\theta_{0}$ sufficiently close to $\pi / 4, g\left(\theta_{0}\right)$ is positive and so $r^{\prime}\left(\theta_{0}\right)>r\left(\theta_{0}\right)$. However $p$ and $q$ need not be of the same sign. Thus there are $q$, positive but small enough, such that we can select a negative $p$ which satisfies $p>(4 / \pi)^{q+1}-2$. For such $p, q$ and for $\theta_{0}$ sufficiently close to $\pi / 4$, (6.7) is again reversed.

It seems quite likely that the restriction on $p$ in (6.7) and on $q$ in (6.6) cannot be lightened.

From (6.7) we can prove a Brunn-Minkowski type theorem. Let $\mathbf{K}$ be a bounded, measurable family of convex bodies in $R_{n}$ over $T$. We form the family $\mathbf{C}$ over $T \times \mathscr{R}_{n} \times \mathscr{R}_{n-p}^{\prime}$ defined by $C\left(t, \rho, \rho^{\prime}\right)=\rho^{\prime} \cdot K(t) / \rho \cdot L_{n-p}$ in accordance with the notation in the last part of $\S 4$. For $p=0,1, \ldots, n-1$ we have by (4.5)

$$
M_{q}^{t}\left(\left[W_{p}(K(t)) / \kappa_{n}\right]^{1 /(n-p)}\right) E_{n-p}=\dot{M}_{q}^{t} \dot{M}_{n-p}^{\rho} \dot{M}_{n-p}^{\rho^{\prime}}(\mathbf{C}),
$$

in virtue of property (VIb). Suppose $q \leqq-1$; then we may apply (6.7) twice to obtain

$$
\dot{M}_{q}^{t} \dot{M}_{n-p}^{o} \dot{M}_{n-p}^{\rho^{\prime}}(\mathbf{C}) \supseteq \dot{M}_{n-p}^{\rho} \dot{M}_{n-p}^{\rho^{\prime}} \dot{M}_{q}^{t}(\mathbf{C}) .
$$

We next use (5.12) and the fact that

$$
\dot{M}_{q}^{t}\left(\rho^{\prime} \cdot K(t)\right)=\rho^{\prime} \cdot \dot{M}_{q}^{t}(K(t))
$$

to get

$$
\dot{M}_{n-p}^{\rho} \dot{M}_{n-p}^{\rho^{\prime}} \dot{M}_{q}^{t}(\mathbf{C}) \supseteq \dot{M}_{n-p}^{\rho} \dot{M}_{n-p}^{\rho^{\prime}}\left(\rho^{\prime} \cdot \dot{M}_{q}^{t}(K(t)) / \rho \cdot L_{n-p}\right) .
$$

Again by (4.5) the last expression is $\left[W_{p}\left(\dot{M}_{q}^{t}(K(t))\right) / \kappa_{n}\right]^{1 /(n-p)} E_{n-p}$. Hence (6.8) and (6.9) yield

$$
W_{p}^{1 /(n-p)}\left(\dot{M}_{q}^{t}(K(t))\right) \leqq M_{q}^{t}\left(W_{p}^{1 /(n-p)}(K(t))\right), \quad q \leqq-1 .
$$

This inequality was proved, less systematically, in [5], [6], and [7]; the cases of equality can be found there. 
7. We define a system of power means to be a collection of power means $\left\{N_{p}\right\}$ of families of convex bodies, one for each index $p$ satisfying $-\infty<p<\infty$, which are continuously and monotonically dependent on $p$. We have

$$
\lim _{p \rightarrow-\infty} N_{p}(\mathbf{K})=\gamma(\mathbf{K}), \quad \lim _{p \rightarrow \infty} N_{p}(\mathbf{K})=\Gamma(\mathbf{K})
$$

in virtue of Theorem 8 and the corresponding limit relations (6.4) for $M_{p}$ and $\dot{M}_{p}$. Therefore $N_{p}$ is monotonic increasing in $p$, (in the wide sense), and so fulfills Jensen's inequality

$$
N_{p}(\mathbf{K}) \subseteq N_{q}(\mathbf{K}) \quad \text { when } p \leqq q .
$$

We have seen that the collections $\left\{M_{p}\right\}$ and $\left\{\dot{M}_{p}\right\}$ each form a system of power means. These are not the only possible systems however; we shall construct other systems of power means in this section. At the same time, two collections of power means will be displayed which satisfy all the requirements for a system except that in one case Jensen's inequality is not fulfilled and in the other case the continuity assumption is not satisfied. This will show the independence of these assumptions.

Let us begin with a comment on inequality (5.7): there are cases of strict inequality. To see this, take $T=T_{1}, p=0$, and

$$
K(t)=K \text { for } 0 \leqq t<\frac{1}{2}, \quad K(t)=\hat{K} \text { for } \frac{1}{2} \leqq t \leqq 1 .
$$

For this family (5.7) reads

$$
\dot{M}_{0}\left(K, \hat{K} ; \frac{1}{2}\right) \subseteq M_{0}\left(K, \hat{K} ; \frac{1}{2}\right) .
$$

Suppose there is equality for all convex bodies $K$; then

$$
\dot{M}_{0}\left(K, \hat{K} ; \frac{1}{2}\right)=\dot{M}_{0}\left(\hat{K}, K ; \frac{1}{2}\right)=M_{0}\left(\hat{K}, K ; \frac{1}{2}\right)=\left[\dot{M}_{0}\left(K, \hat{K} ; \frac{1}{2}\right)\right]^{\wedge}
$$

by the symmetry property (III) of $\dot{M}_{0}$ and (3.3). This equality asserts that $\dot{M}_{0}\left(K, \hat{K} ; \frac{1}{2}\right)$ is always its own polar reciprocal with respect to $E$. But $E$ is the only convex body with this property. Now if $H$ and $F$ are the support and distance functions of $K$, then $\dot{M}_{0}\left(K, \hat{K} ; \frac{1}{2}\right)$ is the convex closure of that star body whose distance function is $(H F)^{1 / 2}$. On the other hand, the only star body which has $E$ as its convex closure is $E$ itself. Hence we would have to have, for any convex body $K$,

$$
(H(x) F(x))^{1 / 2}=\|x\| \text { for all } x \text { in } R_{n} .
$$

For a specific counterexample, take $K$ to be the cube of points $x$ for which $F(x)=\max \left\{\left|x^{1}\right|, \ldots,\left|x^{n}\right|\right\} \leqq 1$ and, for this choice of $K, H(x)=\left|x^{1}\right|+\cdots+\left|x^{n}\right|$. Therefore there are cases of inequality in (5.7). The continuity of $M_{p}$ and $\dot{M}_{p}$ in $p$ and $\mathbf{K}$ shows that this is not an isolated instance.

The result may be viewed in another light. Let $f$ be any positive, measurable, bounded function over $T$ which is bounded away from zero. Then the function $\hat{f}$ defined over $T$ by $\hat{f}(t)=1 / f(t)$ is also of this character and we have

$$
\hat{M}_{p}(\hat{f})=M_{-p}(f)
$$


where, for $a>0, \hat{a}=1 / a$. Thus the geometric mean of real-valued functions may be said to be self-conjugate. As we have seen (7.2) is not true for the power means $M_{p}$ or $\dot{M}_{p}$ of families of convex bodies. Several of the systems of means constructed in this section will have the analogue of property (7.2).

Let $\Theta$ be an infinite sequence of real numbers $\theta_{0}, \theta_{1}, \ldots, \theta_{k}, \ldots$ which satisfy $0 \leqq \theta_{j} \leqq 1$ and suppose $\Theta$ has a limit $\theta$. Further take $\mathbf{K}$ to be a bounded and measurable family of convex bodies over $T$. Set

$$
C_{0}=M_{p}(\mathbf{K}), \quad C_{0}^{\prime}=\dot{M}_{p}(\mathbf{K})
$$

and define inductively

$$
C_{j}=M_{p}\left(C_{j-1}, C_{j-1}^{\prime} ; \theta_{j-1}\right), \quad C_{j}^{\prime}=\dot{M}_{p}\left(C_{j-1}, C_{j-1}^{\prime} ; \theta_{j-1}\right)
$$

for $j=1,2, \ldots$

From (5.7) we have $C_{j}^{\prime} \subseteq C_{j}$; therefore

$$
\begin{aligned}
& C_{j}=M_{p}\left(C_{j-1}, C_{j-1}^{\prime} ; \theta_{j-1}\right) \subseteq M_{p}\left(C_{j-1} C_{j-1} ; \theta_{j-1}\right)=C_{j-1}, \\
& C_{j}^{\prime}=\dot{M}_{p}\left(C_{j-1}, C_{j-1}^{\prime} ; \theta_{j-1}\right) \supseteq \dot{M}_{p}\left(C_{j-1}^{\prime}, C_{j-1}^{\prime} ; \theta_{j-1}\right)=C_{j-1}^{\prime}
\end{aligned}
$$

by properties (II) and (IV) in its generalized form following (5.19). To summarize:

$$
C_{j-1}^{\prime} \subseteq C_{j}^{\prime} \subseteq C_{j} \subseteq C_{j-1} .
$$

The two sequences $\left\{C_{j}^{\prime}\right\},\left\{C_{j}\right\}$ of convex bodies are monotonic and bounded by $C_{0}, C_{0}^{\prime}$. Hence they have limits $C^{\prime}$ and $C$ respectively which satisfy

$$
C_{0}^{\prime} \subseteq C^{\prime} \subseteq C \subseteq C_{0},
$$

cf. [10, p. 152]. Let $F^{\prime}, F$ be the distance functions of $C^{\prime}, C$.

If $\theta_{m}$ is one for some index $m$, we conclude from (7.3) and the generalized property (IV) as well as (7.4), that all the subsequent members of the sequences $\left\{C_{j}\right\},\left\{C_{j}^{\prime}\right\}$ are just $C_{m}^{\prime}$. In this case $C=C^{\prime}=C_{m}^{\prime}$.

In a similar fashion, if $\theta_{m}$ is zero for some index $m, C=C^{\prime}=C_{m}$.

Next suppose that $0<\theta_{j}<1$ for all $j$ and that $\theta \neq 1$. From the continuity of $\dot{M}_{p}\left(K_{0}, K_{1} ; \vartheta\right)$ in $\left(K_{0}, K_{1} ; \vartheta\right)$, we deduce

$$
\lim _{j \rightarrow \infty} \dot{M}_{p}\left(C_{j-1}, C_{j-1}^{\prime} ; \theta_{j-1}\right)=\dot{M}_{p}\left(C, C^{\prime \prime} ; \theta\right)=C^{\prime} .
$$

Therefore $M_{-p}\left(F, F^{\prime} ; \theta\right)$ is the distance function of a star body contained in $C^{\prime}$, and so $F^{\prime}(x) \leqq M_{-p}\left(F(x), F^{\prime}(x) ; \theta\right)$. Since $\theta \neq 1$, this implies that $F^{\prime}(x) \leqq F(x)$ over $R_{n}$ which, taken together with (7.5), gives $C^{\prime}=C$.

If $\theta=1$, then $C=M_{p}\left(C, C^{\prime} ; 1\right)=C^{\prime}$ as before.

In all these cases we denote the common limit $C$ by $N_{p}(\mathbf{K} \mid \Theta)$. When $\theta_{j}=\theta$ for all $j$ we will write the number $\theta$ for the sequential symbol $\Theta$.

The principal result in this section is the following theorem.

THEOREM 9. The collection $\left\{N_{p}(\mathbf{K} \mid \Theta)\right\}$ forms a system of power means for each sequence $\Theta$ of the type allowed, i.e., for each real, finite $p, N_{p}(\mathbf{K} \mid \Theta)$ possesses 
properties (I) through (VIb) and, for each $\mathbf{K}, N_{p}(\mathbf{K} \mid \Theta)$ is continuous and monotonic in $p$.

First we demonstrate properties (I) through (VIb).

(I) Suppose $\left\{\mathbf{K}_{j}\right\}$ is a sequence of families in $D_{n}$ which converges to a family $\mathbf{K}$ in $D_{n}$ over $T_{1}$. By the continuity of $M_{p}$ and $\dot{M}_{p}$, we know that the sequences

$$
\left\{M_{p}\left(\mathbf{K}_{j}\right)\right\}=\left\{C_{0}^{(j)}\right\} \text { and }\left\{\dot{M}_{p}\left(\mathbf{K}_{j}\right)\right\}=\left\{C_{0}^{\prime(j)}\right\}
$$

converge to $C_{0}=M_{p}(\mathbf{K})$ and $C_{0}^{\prime}=\dot{M}_{p}(\mathbf{K})$ respectively. From this we next prove that, given $\varepsilon$ positive and less than one, there is an $n(\varepsilon)$ for which

$$
(1+\varepsilon) C_{0} \supseteq C_{0}^{(j)} ; \quad(1-\varepsilon) C_{0} \subseteq C_{0}^{(j)} ; \quad(1+\varepsilon) C_{0}^{\prime} \supseteq C_{0}^{(j)} ; \quad(1-\varepsilon) C_{0}^{\prime} \subseteq C_{0}^{(j)},
$$

whenever $j>n(\varepsilon)$.

Consider the first pair of (7.6). The convergence of $\left\{C_{0}^{(j)}\right\}$ to $C_{0}$ implies the existence of an $n_{0}(\varepsilon)$ such that, for $j>n_{0}(\varepsilon)$, we have $\delta\left(C_{0}, C_{0}^{(j)}\right)<\varepsilon$ i.e., $C_{0}+\varepsilon E \supseteq C_{0}^{(j)}$, $C_{0}^{(j)}+\varepsilon E \supseteq C_{0}$. Since $b_{0} E \subseteq K_{j}(t) \subseteq b_{1} E, b_{0} E \subseteq K(t) \subseteq b_{1} E$ for some positive $b_{0}, b_{1}$, we deduce $b_{0} E \subseteq C_{0}$ and so $\left(1+\varepsilon / b_{0}\right) C_{0} \supseteq C_{0}^{(j)}$. Similarly from $b_{0} E \subseteq C_{0}^{(j)}$, we obtain $\left(1+\varepsilon / b_{0}\right) C_{0}^{(j)} \supseteq C_{0}$. From this last inequality we have

$$
C_{0}^{(j)} \supseteq\left(1-\varepsilon /\left[b_{0}+\varepsilon\right]\right) C_{0} \supseteq\left(1-\varepsilon / b_{0}\right) C_{0} .
$$

Hence, for $j>n_{0}\left(b_{0} \varepsilon\right)$ we have the first pair of inequalities in (7.6).

The second pair is treated similarly and holds for $j>n_{0}\left(\varepsilon / b_{1}\right)$; finally take $n(\varepsilon)=\max \left\{n_{0}\left(b_{0} \varepsilon\right), n_{0}\left(\varepsilon / b_{1}\right)\right\}$.

By an inductive argument, it follows that

$$
(1+\varepsilon) C_{k}=M_{p}\left((1+\varepsilon) C_{k-1},(1+\varepsilon) C_{k-1}^{\prime} ; \theta_{k-1}\right) \supseteq M_{p}\left(C_{k-1}^{(j)}, C_{k-1}^{\prime(j)} ; \theta_{k-1}\right)=C_{k}^{(j)}
$$

and, in like fashion,

$$
(1-\varepsilon) C_{k} \subseteq C_{k}^{(j)}, \quad(1+\varepsilon) C_{k}^{\prime} \supseteq C_{k}^{(j)}, \quad(1-\varepsilon) C_{k}^{\prime} \subseteq C_{k}^{(j)},
$$

for $k=1,2, \ldots$, from the set monotonicity of $M_{p}$ and $\dot{M}_{p}$. Consequently, for $j>n(\varepsilon)$

Therefore

$$
(1-\varepsilon) C \subseteq C^{(j)}=\lim _{k \rightarrow \infty} C_{k}^{(j)}, \quad(1+\varepsilon) C \supseteq C^{(j)}
$$

$$
\lim _{j \rightarrow \infty} C^{(j)}=\lim _{j \rightarrow \infty} N_{p}\left(\mathbf{K}_{j} \mid \Theta\right)=C=N_{p}(\mathbf{K} \mid \Theta)
$$

as was to be shown.

(II) For the set monotonicity of $N_{p}$ suppose $K_{1}(t) \subseteq K_{2}(t)$ over $T_{1}$. Then, from the monotonicity of $M_{p}$ and $\dot{M}_{p}$, we have

$$
C_{0}^{(1)}=M_{p}\left(\mathbf{K}_{1}\right) \subseteq C_{0}^{(2)}=M_{p}\left(\mathbf{K}_{2}\right), \quad C_{0}^{\prime(1)}=\dot{M}_{p}\left(\mathbf{K}_{1}\right) \subseteq C_{0}^{\prime(2)}=\dot{M}_{p}\left(\mathbf{K}_{2}\right) .
$$

By the obvious induction argument, we conclude that $C_{j}^{(1)} \subseteq C_{j}^{(2)}, C_{j}^{\prime(1)} \subseteq C_{j}^{\prime(2)}$, for all $j$, whence

$$
C^{(1)}=N_{p}\left(\mathbf{K}_{1} \mid \Theta\right) \subseteq C^{(2)}=N_{p}\left(\mathbf{K}_{2} \mid \Theta\right)
$$


(III) and (V) both follow from the fact that the sequences $\left\{C_{j}\right\},\left\{C_{j}^{\prime}\right\}$ generated from $\mathbf{K}$ are equal, term by term to those generated from $\mathbf{K}^{\prime}$.

(IV) All the elements of $\left\{C_{k}\right\},\left\{C_{k}^{\prime}\right\}$ are $E$; hence so is $N_{p}(\mathbf{K} \mid \Theta)$.

(VIa) From $M_{p}(\lambda \mathbf{K})=\lambda C_{0}, \dot{M}_{p}(\lambda \mathbf{K})=\lambda C_{0}^{\prime}$, the property follows by an inductive argument.

(VIb) From

$$
M_{p}(\lambda(t) K)=\mu C_{0}, \quad \dot{M}_{p}(\lambda(t) K)=\mu C_{0}^{\prime}
$$

we have the same conclusion as in (VIb).

For the monotonicity in $p$ we demonstrate Jensen's inequality

$$
N_{p}(\mathbf{K} \mid \Theta) \subseteq N_{q}(\mathbf{K} \mid \Theta) \text { when } p<q .
$$

By (6.1), for $p<q$

$$
C_{0}^{\prime}(p)=\dot{M}_{p}(\mathbf{K}) \subseteq \dot{M}_{q}(\mathbf{K})=C_{0}^{\prime}(q) \quad \text { and } \quad C_{0}(p)=M_{p}(\mathbf{K}) \subseteq M_{q}(\mathbf{K})=C_{0}(q) .
$$

Hence

$$
\begin{aligned}
C_{1}^{\prime}(p) & =\dot{M}_{p}\left(C_{0}(p), C_{0}^{\prime}(p) ; \theta_{0}\right) \subseteq \dot{M}_{q}\left(C_{0}(p), C_{0}^{\prime}(p) ; \theta_{0}\right) \\
& \subseteq \dot{M}_{q}\left(C_{0}(q), C_{0}^{\prime}(q) ; \theta_{0}\right)=C_{1}^{\prime}(q),
\end{aligned}
$$

and similarly $C_{1}(p) \subseteq C_{1}(q)$. In this way we establish inductively

$$
C_{j}^{\prime}(p) \subseteq C_{j}^{\prime}(q) \text { and } C_{j}(p) \subseteq C_{j}(q), j=1,2, \ldots
$$

Consequently in the limit we obtain (7.7).

The continuity of $C(p)=N_{p}(\mathbf{K} \mid \Theta)$ is left. First note from the definition (2.11) of $\delta$ that, if $K^{\prime \prime} \subseteq K^{\prime} \subseteq K$ are three convex bodies, then

$$
\delta\left(K^{\prime}, K\right) \leqq \delta\left(K^{\prime \prime}, K\right) \text {. }
$$

Let $p$ be a given real number and suppose $\varepsilon>0$ to be preassigned. Since $\left\{C_{j}(p)\right\}$ and $\left\{C_{j}^{\prime}(p)\right\}$ converge to $C(p)$, there is an index $k$ such that

$$
\delta\left(C_{k}(p), C_{k}^{\prime}(p)\right)<\varepsilon / 5 .
$$

Hence, by (7.8) and (7.4),

$$
\delta\left(C(p), C_{k}(p)\right)<\varepsilon / 5 .
$$

Next, we have already seen that $M_{p}(\mathbf{K})$ and $\dot{M}_{p}(\mathbf{K})$ are continuous in $p$ and, in the same discussion, that the corresponding elementary means $M_{p}\left(K_{0}, K_{1} ; \vartheta\right)$, $\dot{M}_{p}\left(K_{0}, K_{1} ; \vartheta\right)$ are continuous in $\left(p, K_{0}, K_{1}, \vartheta\right)$; this permits us to conclude inductively that $C_{k}(p), C_{k}^{\prime}(p)$ are continuous in $p$ and so we may choose an index $q_{0}>p$ such that:

$$
\delta\left(C_{k}(p), C_{k}\left(q_{0}\right)\right)<\varepsilon / 5, \quad \delta\left(C_{k}^{\prime}(p), C_{k}^{\prime}\left(q_{0}\right)\right)<\varepsilon / 5 .
$$

From this and the monotonicity of $C_{k}(p), C_{k}^{\prime}(p)$ in $p$, we have by (7.8):

$$
\delta\left(C_{k}(p), C_{k}(q)\right)<\varepsilon / 5, \quad \delta\left(C_{k}^{\prime}(p), C_{k}^{\prime}(q)\right)<\varepsilon / 5,
$$


for all $q$ satisfying $p<q \leqq q_{0}$. By (7.9), (7.11), and the triangle inequality for $\delta$ we obtain, for the same $q, \delta\left(C_{k}(q), C_{k}^{\prime}(q)\right)<3 \varepsilon / 5$. With the same argument that gave (7.10) we deduce

$$
\delta\left(C(q), C_{k}(q)\right)<3 \varepsilon / 5 .
$$

By the triangle inequality and (7.10), the first part of (7.11), and (7.12) we get finally

$$
\delta(C(p), C(q))<\varepsilon
$$

for all $q$ satisfying $p<q \leqq q_{0}$. In the same way, there can be found a $q_{0}^{\prime}<p$ such that, for all $q$ satisfying $q_{0}^{\prime} \leqq q<p$, we have (7.13); from this we have the desired continuity.

With some modifications the preceding result can be extended to cover the following situation: we suppose $\Theta$ to be a sequence of nonincreasing functions of $p$ which are equicontinuous at each finite $p$ and converge to a continuous limit function. Then $N_{p}(\mathbf{K} \mid \Theta(p))$ is continuous in $p$. The only alterations in the argument come in the inductive proof that $C_{k}(p), C_{k}^{\prime}(p)$ are continuous in $p$ and in the demonstration that these sets are monotonic in $p$. For the first the equicontinuity of $\Theta$ is needed; for the second, the monotonic character of the elements of $\Theta$.

From (7.5), and in harmony with Theorem 8, we have

$$
\dot{M}_{p}(\mathbf{K})=N_{p}(\mathbf{K} \mid 1) \subseteq N_{p}(\mathbf{K} \mid \Theta) \subseteq N_{p}(\mathbf{K} \mid 0)=M_{p}(\mathbf{K}) .
$$

These inequalities can be generalized considerably. We write $\Theta \leqq \Theta^{\prime}$ if $\theta_{j}^{\prime}-\theta_{j}$ is nonnegative for all $j$. The generalization is this: if $\Theta \leqq \Theta^{\prime}$ then

$$
N_{p}\left(\mathbf{K} \mid \Theta^{\prime}\right) \subseteq N_{p}(\mathbf{K} \mid \Theta) \text {. }
$$

As a preliminary we remark that if $K_{0}$ and $K_{1}$ are two convex bodies such that $K_{0} \supseteq K_{1}$ then the elementary means $M_{p}\left(K_{0}, K_{1} ; \vartheta\right), \dot{M}_{p}\left(K_{0}, K_{1} ; \vartheta\right)$ decrease monotonically from $K_{0}$ to $K_{1}$ as $\vartheta$ goes from 0 to 1 . This is an immediate consequence of property (II) with the use of piecewise constant families $\mathbf{K}$ defined by

$$
K(t)=K_{0} \quad \text { for } 0 \leqq t \leqq \vartheta, \quad K(t)=K_{1} \quad \text { for } \vartheta<t \leqq 1 .
$$

To form the right and left sides of (7.14) we begin in both cases with $C_{0}=M_{p}(\mathbf{K})$ $\supseteq \dot{M}_{p}(\mathbf{K})=C_{0}^{\prime}$, and next form

$$
C_{1}\left(\theta_{0}\right)=M_{p}\left(C_{0}, C_{0}^{\prime} ; \theta_{0}\right), \quad C_{1}^{\prime}\left(\theta_{0}\right)=\dot{M}_{p}\left(C_{0}, C_{0}^{\prime} ; \theta_{0}\right)
$$

and, similarly, $C_{1}\left(\theta_{0}^{\prime}\right), C_{1}^{\prime}\left(\theta_{0}^{\prime}\right)$. Since $\theta_{0} \leqq \theta_{0}^{\prime}$ by the monotonicity property just mentioned

$$
C_{1}\left(\theta_{0}\right) \supseteq C_{1}\left(\theta_{0}^{\prime}\right), \quad C_{1}^{\prime}\left(\theta_{0}\right) \supseteq C_{1}^{\prime}\left(\theta_{0}^{\prime}\right) .
$$

We now form

$$
C_{2}\left(\theta_{0}, \theta_{1}\right)=M_{p}\left(C_{1}\left(\theta_{0}\right), C_{1}^{\prime}\left(\theta_{0}\right) ; \theta_{1}\right), \quad C_{2}^{\prime}\left(\theta_{0}, \theta_{1}\right)=\dot{M}_{p}\left(C_{1}\left(\theta_{0}\right), C_{1}^{\prime}\left(\theta_{0}\right) ; \theta_{1}\right)
$$


and similarly $C_{2}\left(\theta_{0}^{\prime}, \theta_{1}\right), C_{2}^{\prime}\left(\theta_{0}^{\prime}, \theta_{1}\right), C_{2}\left(\theta_{0}^{\prime}, \theta_{1}^{\prime}\right), C_{2}^{\prime}\left(\theta_{0}^{\prime}, \theta_{1}^{\prime}\right)$. From property (III) and the set monotonicity in the parameter $\vartheta$ we obtain

$$
C_{2}\left(\theta_{0}, \theta_{1}\right) \supseteq C_{2}\left(\theta_{0}^{\prime}, \theta_{1}\right) \supseteq C_{2}\left(\theta_{0}^{\prime}, \theta_{1}^{\prime}\right), \quad C_{2}^{\prime}\left(\theta_{0}, \theta_{1}\right) \supseteq C_{2}^{\prime}\left(\theta_{0}^{\prime}, \theta_{1}\right) \supseteq C_{2}^{\prime}\left(\theta_{0}^{\prime}, \theta_{1}^{\prime}\right)
$$

since $\theta_{1} \leqq \theta_{1}^{\prime}$. The proof of (7.14) can be completed inductively.

Consider the sequences $\left\{\hat{C}_{j}^{\prime}\right\},\left\{\hat{C}_{j}\right\}$. We have

$$
\begin{aligned}
\hat{C}_{j}^{\prime}=\left[\dot{M}_{p}\left(C_{j-1}, C_{j-1}^{\prime} ; \theta_{j-1}\right]^{\wedge}\right. & =M_{-p}\left(\hat{C}_{j-1}, \hat{C}_{j-1}^{\prime} ; \theta_{j-1}\right) \\
& =M_{-p}\left(\hat{C}_{j-1}^{\prime}, \hat{C}_{j-1} ; 1-\theta_{j-1}\right)
\end{aligned}
$$

from (3.3). In the same way $\hat{C}_{j}=\dot{M}_{-p}\left(\hat{C}_{j-1}^{\prime}, \hat{C}_{j-1} ; 1-\theta_{j-1}\right)$. The limit of these sequences is seen to be the result of joint iteration using the means $M_{-p}$ and $\dot{M}_{-p}$ together with the sequence $\hat{\Theta}: 1-\theta_{0}, 1-\theta_{1}, \ldots, 1-\theta_{j}, \ldots$, and beginning with the means

$$
\hat{C}_{0}^{\prime}=\left[\dot{M}_{p}(\mathbf{K})\right]^{\wedge}=M_{-p}(\hat{\mathbf{K}}), \quad \hat{C}_{0}=\left[M_{p}(\mathbf{K})\right]^{\wedge}=\dot{M}_{-p}(\hat{\mathbf{K}}) .
$$

Thus the common limit is $N_{-p}(\hat{\mathbf{K}} \mid \hat{\Theta})$.

On the other hand $\lim _{j \rightarrow \infty} \hat{C}_{j}=\hat{C}=\hat{N}_{p}(\mathbf{K} \mid \Theta)$ which gives $\hat{N}_{p}(\mathbf{K} \mid \Theta)=N_{-p}(\hat{\mathbf{K}} \mid \Theta)$ or, finally, by taking polar reciprocals,

$$
N_{p}(\mathbf{K} \mid \Theta)=\hat{N}_{-p}(\hat{\mathbf{K}} \mid \Theta \hat{)} \text {. }
$$

We call a system of means $\left\{N_{p}(\mathbf{K})\right\}$ self-conjugate if for each $p$ and each bounded and measurable family $\mathbf{K}$ of convex bodies over $T: N_{p}(\mathbf{K})=\hat{N}_{-p}(\hat{\mathbf{K}})$. To construct some self-conjugate systems, let $\left\{\theta_{j}(p)\right\}=\Theta(p)$ be a sequence of nonnegative, monotonic, nonincreasing functions which are equicontinuous over any finite $p$-interval. We suppose that the sequence converges to a continuous limit $\theta(p)$ and that

$$
\theta_{j}(p)+\theta_{j}(-p)=\theta(p)+\theta(-p)=1 .
$$

It is easily seen that for each $p$ the sequence is of the type allowed in the formation of $N_{p}(\mathbf{K} \mid \Theta)$; set ${ }_{1} N_{p}(\mathbf{K})=N_{p}(\mathbf{K} \mid \Theta(p))$. Thus for each $p,{ }_{1} N_{p}(\mathbf{K})$ is a mean and by our extended continuity property, the collection $\left\{{ }_{1} N_{p}(\mathbf{K})\right\}$ depends continuously on $p$.

To prove Jensen's inequality we apply (7.7) and (7.8): for $p<q$ we find

$$
{ }_{1} N_{p}(\mathbf{K})=N_{p}(\mathbf{K} \mid \Theta(p)) \subseteq N_{p}(\mathbf{K} \mid \Theta(q)) \subseteq N_{q}(\mathbf{K} \mid \Theta(q))={ }_{1} N_{q}(\mathbf{K})
$$

since the elements of $\Theta(p)$ are nonincreasing in $p$.

Hence $\left\{{ }_{1} N_{p}(\mathbf{K})\right\}$ is a system. By (7.15)

$$
{ }_{1} \hat{N}_{-p}(\hat{\mathbf{K}})=\hat{N}_{-p}(\hat{\mathbf{K}} \mid \Theta(-p))=N_{p}(\mathbf{K} \mid \Theta(-p))=N_{p}(\mathbf{K} \mid \Theta(p))={ }_{1} N_{p}(\mathbf{K}) .
$$

The next to the last equality comes from (7.16) and the definition of $\Theta$. Thus $\left\{{ }_{1} N_{p}(\mathbf{K})\right\}$ is a self-conjugate system.

When $p=0$, (7.16) shows that $\theta_{j}(0)=\frac{1}{2}$. Hence if $\mathbf{K}$ is the family described by (7.1)

$$
{ }_{1} N_{0}(\mathbf{K})=N_{0}\left(\mathbf{K} \mid \frac{1}{2}\right)=\hat{N}_{0}\left(\mathbf{K} \mid \frac{1}{2}\right)=E
$$


since the convex body is self-polar. Here we have used the symmetry property of ${ }_{1} N_{0}$. Thus, for this system, the geometric mean of a convex body and its polar reciprocal is the unit sphere.

We close with two examples of collections of means which are not systems. The first, $\left\{{ }_{1} M_{p}(\mathbf{K})\right\}$ satisfies all the requirements excepting continuity in $p$; the second, $\left\{{ }_{2} M_{p}(\mathbf{K})\right\}$ satisfies all the requirements excepting Jensen's inequality is not always fulfilled. We set

$$
{ }_{i} M_{p}(\mathbf{K})=M_{p}\left(M_{p}(\mathbf{K}), \dot{M}_{p}(\mathbf{K}) ; \vartheta_{i}(p)\right), \quad i=1,2 .
$$

For $\vartheta_{1}$ we choose $\vartheta_{1}(p)=1$ for $p<0, \vartheta_{1}(p)=0$ for $p \geqq 0$.

We have noted at the beginning of this section that there are families $\mathbf{K}$ for which

$$
\dot{M}_{0}(\mathbf{K}) \subset M_{0}(\mathbf{K}) .
$$

Then for $q_{0}>0$, but small enough, we have $\dot{M}_{q_{0}}(\mathbf{K}) \nsubseteq M_{0}(\mathbf{K})$ in virtue of the continuity of $\dot{M}_{q}$ in $q$. Let $\vartheta_{2}(p)=0$ for $p<0, \vartheta_{2}(p)=p / q_{0}$ for $0 \leqq p \leqq q_{0}, \vartheta_{2}(p)=1$ for $p>q_{0}$. From the foregoing discussions $\left\{{ }_{2} M_{p}(\mathbf{K})\right\}$ is a collection of means which depend continuously on $p$. However, for the family satisfying (7.17):

$$
{ }_{2} M_{0}(\mathbf{K})=M_{0}(\mathbf{K}) \nsubseteq \dot{M}_{q_{0}}(\mathbf{K})={ }_{2} M_{q_{0}}(\mathbf{K}) \text {. }
$$

\section{REFERENCES}

1. T. Bonnesen and W. Fenchel, Theorie der konvexen Körper, Springer, Berlin, 1934.

2. A. Dinghas, Zum Minkowskischen Integralbegriff abgeschlossener Mengen, Math. Z. 66 (1956), 173-188.

3. -_- Minkowskische Summen und Integrale, superadditive Mengenfunktionale, isoperimetrische Ungleichungen, Gauthier-Villars, Paris, 1961.

4. W. Firey, p-means of convex bodies, Math. Scand. 10 (1962), 17-24.

5. - Polar means of convex bodies and a dual to the Brunn-Minkowski theorem, Canad. J. Math. 13 (1961), 444-453.

6. - Mean cross-section measures of harmonic means of convex bodies, Pacific J. Math. 11 (1961), 1263-1266.

7. - Some applications of means of convex bodies, Pacific J. Math. 14 (1964), 53-60.

8. P. Halmos, Measure theory, Van Nostrand, New York, 1950.

9. G. Hardy, J. Littlewood and G. Pólya, Inequalities, Cambridge Univ. Press, Cambridge, 1934.

10. H. Hadwiger, Vorlesungen über Inhalt, Oberfläche und Isoperimetrie, Springer, Berlin, 1957.

11. A. Kolmogoroff, Sur la notion de la moyenne, Atti Accad. Naz. Lincei (6) 12 (1930), 388-391.

12. J. L. W. V. Jensen, Om konvekse Funktioner og Uligheder imellem Middelvaerdier, Nyt Tidsskrift Mat. B 16 (1905), 49-68.

13. B. Jessen, Bemaerkninger om konvekse Funktioner og Uligheder imellem Middelvaerdier. I, II, Mat. Tidsskrift B (1931), 17-28; 84-95.

14. M. Nagumo, Über eine Klasse der Mittelwerte, Japan. J. Math. 7 (1930), 71-79.

Oregon State University, CORVALLis, OREGON 